Research Article

\title{
Comprehensive Bioinformatics Analysis of Lipopolysaccharide- Induced Altered Autophagy in Acute Lung Injury and Construction of Underlying Competing Endogenous RNA Regulatory Mechanism
}

\author{
Jian-Yu Liu $\left(\mathbb{D},{ }^{1}\right.$ Ying-Xiao Jiang, ${ }^{1}$ Meng-Yu Zhang, ${ }^{1}$ Chen Huo, ${ }^{1}$ Yi-Can Yang, ${ }^{1}$ Xiu-Li Ji, \\ and Yi-Qing $\mathbf{Q u} \mathbb{1}^{3}$ \\ ${ }^{1}$ Department of Pulmonary and Critical Care Medicine, Qilu Hospital, Cheeloo College of Medicine, Shandong University, \\ Shandong Key Laboratory of Infectious Respiratory Diseases, China \\ ${ }^{2}$ Department of Pulmonary Disease, Jinan Traditional Chinese Medicine Hospital, China \\ ${ }^{3}$ Department of Pulmonary and Critical Care Medicine, Qilu Hospital of Shandong University, \\ Shandong Key Laboratory of Infectious Respiratory Disease, China
}

Correspondence should be addressed to Yi-Qing Qu; quyiqing@sdu.edu.cn

Received 15 July 2021; Revised 18 August 2021; Accepted 18 September 2021; Published 21 October 2021

Academic Editor: Nagarajan Raju

Copyright (c) 2021 Jian-Yu Liu et al. This is an open access article distributed under the Creative Commons Attribution License, which permits unrestricted use, distribution, and reproduction in any medium, provided the original work is properly cited.

\begin{abstract}
Background. Acute lung injury (ALI) is a fatal syndrome frequently induced by lipopolysaccharide (LPS) released from the bacterial cell wall. LPS could also trigger autophagy of lung bronchial epithelial cell to relieve the inflammation, while the overwhelming LPS would impair the balance of autophagy consequently inducing serious lung injury. Methods. We observed the autophagy variation of $16 \mathrm{HBE}$, human bronchial epithelial cell, under exposure to different concentrations of LPS through western blot, immunofluorescence staining, and electron microscopy. Eight strands of 16HBE were divided into two groups upon $1000 \mathrm{ng} / \mathrm{ml}$ LPS stimulation or not, which were sent to be sequenced at whole transcriptome. Subsequently, we analyzed the sequencing data in functional enrichment, pathway analysis, and candidate gene selection and constructed a hsa-miR-663brelated competing endogenous RNA (ceRNA) network. Results. We set a series of concentrations of LPS to stimulate 16HBE and observed the variation of autophagy in related protein expression and autophagosome count. We found that the effective concentration of LPS was $1000 \mathrm{ng} / \mathrm{ml}$ at 12 hours of exposure and sequenced the $1000 \mathrm{ng} / \mathrm{ml} \mathrm{LPS}$-stimulated 16HBE. As a result, a total of 750 differentially expressed genes (DEGs), 449 differentially expressed lncRNAs (DElncRNAs), 76 differentially expressed circRNAs (DEcircRNAs), and 127 differentially expressed miRNAs (DEmiRNAs) were identified. We constructed the protein-protein interaction (PPI) network to visualize the interaction between DEGs and located 36 genes to comprehend the core discrepancy between LPS-stimulated 16HBE and the negative control group. In combined analysis of differentially expressed RNAs (DERNAs), we analyzed all the targeted relationships of ceRNA in DERNAs and figured hsa-miR-663b as a central mediator in the ceRNA network to play when LPS induced the variation of autophagy in 16HBE. Conclusion. Our research indicated that the hsa-miR-663b-related ceRNA network may contribute to the key regulatory mechanism in LPSinduced changes of autophagy and ALI.
\end{abstract}

\section{Introduction}

ALI, an overwhelming inflammatory response within lung and severely impaired function of gas change $[1,2]$, is a common syndrome with high morbidity and mortality which is induced by endotoxins, complement activation, acid aspiration, and hyperoxia [3]. LPS released from the bacterial cell wall is proved to be a vital incentive for ALI development, which could activate various inflammatory pathways [4-6] and result in imbalance of autophagy [7, 8]. Concerning to researches of lung diseases, LPS could facilitate kinds of cells in lung and disease models of mouse 
to release proinflammatory factors and change in structures of tissue [4, 6, 9-11].

Autophagy is an evolutionarily conserved catabolic process for degrading intracellular component to maintain cellular homeostasis and accommodate to environmental irritation [12]. Within the impaired response caused by LPS, autophagy plays a predominant role in regulatory mechanism and reveals interaction with inflammationrelated signaling pathways. Zeng et al. constructed LPSinduced mouse model of ALI and demonstrated that 4-phenyl butyric acid (4-PBA) could reduce the release of the proinflammatory mediators by inhibiting nuclear factor kappa-B (NF- $\kappa \mathrm{B})$ pathway and decrease autophagy flux in ALI mouse via activation of AKT serine/threonine kinase 1 (AKT)/mammalian target of rapamycin (mTOR) signaling pathway [8]. However, their further studies showed that 3-methyladenine (3-MA), a classic inhibitor of autophagy, increased the endoplasmic reticulum (ER) stress and exacerbated cytotoxicity induced by LPS, which indicated autophagy display a protective effect on the progression of ALI. In mice and MH-S cells, Zhao et al. also demonstrated there is severely impaired function of autophagy in LPSinduced ALI model [13]. The levels of cytokines, including interleukin- (IL-) 6, IL-1 $\beta$, and tumor necrosis factor alpha (TNF- $\alpha$ ), are decreased by application of rapamycin, and cell viability is improved with rescued function of autophagy. Similarly, Zhang et al. investigated the regulatory mechanism of autophagy in LPS-induced pulmonary damage. They elaborated that, in the human pulmonary microvascular endothelial cells (HPMVECs), LPS would lead to higher permeability, lower vitality, and increased lactate dehydrogenase $(\mathrm{LDH})$ release rate, and these changes would be aggravated when inhibiting autophagy. In animal experiments, LPS caused severe pulmonary damage, including hemorrhage, leukocyte infiltration and edema in lung tissue, and high level of proinflammatory cytokines, which was also aggravated by inhibition of autophagy [7]. To further investigate the underlying regulatory mechanism between autophagy and ALI, Nosaka et al. construct the mice with myeloid-specific deletion of the autophagic protein ATG16L1 (Atg16lf $\left.{ }^{\mathrm{l} / \mathrm{l}} L y s M^{\mathrm{Cre}}\right)$. They found that the mice suffered hypoxemia and increased lung permeability with significantly higher level of IL- $1 \beta$, which indicated that autophagy exerted a protective role in suppressing inflammasome activation and production of IL-1 $\beta$ [14]. Except for the researches for discussing autophagy in ALI, autophagy also exerts a crucial role for maintaining homeostasis in other LPS-induced and inflammatory disease model. Kong et al. revealed that LPS decreases the numbers of hepatic autophagosome on the exposure of alcohol, and rapamycin could reverse the ethyl alcohol (EtOH)-LPS-induced liver injury via interaction of Toll-like receptor (TLR4)/lymphocyte antigen 96 (MD2) signaling complex [15]. LPS stimulates Leydig cell in accumulation of oxidative stress and causes turbulence of mitochondria, which is the important influential factor involved in the steroidogenic impairment of Leydig cells [16-18]. Li et al.'s research showed that adrenomedullin (ADM) promotes autophagy of Leydig cells to play a protective role of pyroptosis and cell biological functions in response to the exposure of LPS [19]. They compared the ADM with rapamycin and found the similar effect on the phosphorylation of adenosine $5^{\prime}$-monophosphate- (AMP-) activated protein kinase (AMPK)/mTOR signaling pathway, and the combination of ADM and rapamycin exerts synergistic effect to lessen LPS-induced injury of Leydig cells. The protective effects of autophagy in inflammatory diseases are verified in various cell lines, such as RAW264.7 macrophages [20, 21], BV2 microglial cells [22], adipose-derived stem cells (ADSCs) [23], Caco2 and HT-29 colonic adenoma cells [24], hepatic stellate cells [25], and microglia [26].

ceRNA can bridge the interplay with autophagy and phenotypes of other diseases, especially in cancer and agerelated diseases [27, 28]. In common, ceRNA include partial long noncoding RNAs (lncRNAs) and circular RNAs (circRNAs), which are noncoding RNAs (ncRNAs). MicroRNAs (miRNAs) are another kind of ncRNA and can regulate different physiological and pathological processes by targeting messenger RNA (mRNA). IncRNA and circRNA could competitively sponge with targeted miRNA to exert function of ceRNA to release the targeted mRNA [29, 30]. ceRNA can participate in regulating initiation to maturation progresses of autophagy, which modulate autophagy phagophore initiation through upregulating expression of mTOR, unc-51-like autophagy-activating kinase 1 (ULK1), autophagy-related (ATG) 14L, and Beclin-1. ceRNA also can upregulate ATG3, ATG4, ATG5, ATG7, and ATG12 to influence autophagy phagophore elongation [27]. In acute promyelocytic leukemia (APL)-ascites mouse model and APL cell lines, IncRNA HOTAIRM1 facilitates formation of autophagosome to degrade oncoprotein PML nuclear body scaffold (PML)-retinoic acid receptor alpha (RARA) via targeting miR-20a [31]. In contrast, HOX transcript antisense RNA (HOTAIR) could sponge miR-93 to upregulate ATG12 expression in colorectal cancer cells, which is reported to induce autophagy and decrease radiosensitivity [32]. IncRNA PTENP1 can promote autophagy, as a sponge of miR-17, miR-19b, and miR-20a, by targeting ULK1, ATG7, p62, phosphatase and tensin homolog (PTEN), and $\mathrm{PH}$ domain and leucine-rich repeat protein phosphatase 1 (PHLPP), which could promote progression of hepatocellular carcinoma [33, 34]. Concerning to age-related diseases (ARDs), IncRNAs trigger cellular senescence and the senescence-associated secretory phenotypes (SASPs) [28], which are suggested as the two major contributors to inflammation [35]. NIFK antisense RNA 1 (NIFK-AS1) and colon cancer-associated transcript 1 (CCAT1) sponge miR-148a and miR-146a, respectively, to suppress macrophage M2 polarization and malignant behaviors [36, 37]. IncRNA myocardial infarction-associated transcript (MITA), growth arrest-specific 5 (GAS5), HOTAIR, and urothelial cancerassociated 1 (UCA1) can competitively bind miRNA to promote macrophage $\mathrm{M} 1$ polarization, which induces upregulated level of ROS, proinflammatory cytokines, and matrix metalloproteinase [38-41]. Among the mutual transformation of macrophages, M1 polarization is proinflammatory while M2 polarization is anti-inflammatory [42]. As for the regulatory mechanism between autophagy and inflammation, 
ceRNA also play a crucial role in targeting autophagy to influence the progression of inflammation. In vascular endothelial cells (VECs), Huang et al. found that TGFB2 overlapping transcript 1 (TGFB2-OT1), a lncRNA derived from TGFB2, can regulate autophagy [43]. They demonstrated that LPS significantly upregulated the level of TGFB2-OT1 and further use of mRNA chip assay revealed that miR3960, miR4488, and miR4459 are targets of TGFB2-OT1. TGFB2OT1 sponged miR4488, miR4459, and miR3960 to regulate $\mathrm{N}$-acetyltransferase 8-like (NAT8L), La ribonucleoprotein 1, translational regulator (LARP1), and ceramide synthase 1 (CERS1), which could affect mitochondrial functions by participating in autophagy and induce production of IL-6, IL-8, and IL- $1 \beta$. Our aim is to investigate the regulatory mechanism of ceRNA between autophagy and LPS-induced ALI and find out the key mediators among the ceRNA network.

\section{Material and Methods}

2.1. Cell Culture, Antibodies, and Reagents. Immortalized bronchial epithelial cell line 16HBE cells were obtained from Procell Technology (Wuhan, China). They were cultured in RPMI 1640 (Gibco) at $37^{\circ} \mathrm{C}$ with $5 \% \mathrm{CO}_{2}$ and cocultured with $10 \%$ foetal bovine serum (Biological Industries). According to instruction, we dissolve the LPS powder into different concentrations and stimulate 16HBE for 12 hours.

Antibodies used were rabbit anti-LC3B (Cell Signaling Technology, 3868), rabbit anti-SQSTM1/p62 (Cell Signaling Technology, 8025), and mouse anti-GAPDH (Beyotime, AF5009). Lipopolysaccharide (Sigma-Aldrich, L4391), DAPI (Solarbio, C0065), and RAPA (Sigma-Aldrich, 553210) were used in this study.

2.2. Sample Collection and Preparation. RNA degradation was operated on $1.5 \%$ agarose gels. RNA integrity was assessed using the RNA Nano 6000 Assay Kit (Agilent Technologies, CA, USA). RNA concentration and purity were measured using the NanoDrop 2000 Spectrophotometer (Thermo Fisher Scientific, Wilmington, DE).

The rRNA removal used the Ribo-Zero rRNA Removal Kit (Epicentre, Madison, WI, USA) with $1.5 \mu \mathrm{g}$ RNA per sample. Sequencing libraries were generated using NEBNext $^{R}$ Ultra $^{\mathrm{TM}}$ Directional RNA Library Prep Kit for Illumina ${ }^{\mathrm{R}}$ (NEB, USA) following the manufacturer's recommendations. Finally, PCR products were purified (AMPure XP system) and library quality was assessed on the Agilent Bioanalyzer 2100 and qPCR.

RNA sample preparations consumed $2.5 \mathrm{ng}$ RNA per sample as input material. Sequencing libraries were generated using NEBNext ${ }^{\mathrm{R}}$ Ultra $^{\mathrm{TM}}$ small RNA Sample Library Prep Kit for Illumina ${ }^{\mathrm{R}}$ (NEB, USA) following the manufacturer's recommendations. PCR products were purified, and library quality was assessed as mentioned above.

After cluster generation using TruSeq PE Cluster Kit v3cBot-HS (Illumina), the library preparations were sequenced on an Illumina platform and reads were generated.

2.3. Quantification of Gene Expression Levels and Significant Differentially Expressed RNAs Screening. The levels of gene expression were estimated by fragments per kilobase of transcript per million fragments mapped, i.e., FPKM. The formula is shown as follows:

$\mathrm{FPKM}=\frac{\mathrm{cDNA} \text { fragments }}{\text { Mapped fragments (millions) } \times \text { Transcript length }(\mathrm{kb})}$.

Differential expression analysis was performed using the DESeq $\mathrm{R}$ package. The resulting $P$ values were adjusted using the Benjamini and Hochberg approach for controlling the false discovery rate. Genes with an adjusted $P$ value $<$ 0.01 and absolute value of $\log _{2}$ (fold change) $>1$ found by DESeq were assigned as differentially expressed. The DERNAs, including DElncRNAs, DEmiRNAs, DEcircRNAs, and DEGs, were identified by the limma package in $\mathrm{R}$ which was performed to identify. The ggplot $2 \mathrm{R}$ package was used to generate volcano plot. $\log \mathrm{FC}>0$ indicated that genes were downregulated; in contrast, genes were upregulated with $\log \mathrm{FC}<0$. The significant differentially expressed RNAs were defined as $P<0.05$ and $|\log \mathrm{FC}|>1.5$.

2.4. The Functional Enrichment and Pathway Analysis. Gene Ontology (GO) enrichment analysis of the DEGs was implemented by the clusterProfiler R packages. Enrichment analysis uses hypergeometric testing to find GO entries that are significantly enriched compared to the entire genome background. Gene set enrichment analysis (GSEA) can also be analyzed by clusterProfiler.

Kyoto Encyclopedia of Genes and Genomes (KEGG) is a database resource for comprehending the advanced functions and biosystem in molecular level, which could make relevance between sequenced genomes and systemic functions of cell, species, and ecosystem. We found the significantly enriched KEGG pathway of DEGs via clusterProfiler $\mathrm{R}$ packages compared to the entire genome background.

2.5. Candidate Gene Selection. We used Search Tool for the Retrieval of Interacting Genes (STRING) (http://string-db .org/) to figure out the interaction relationship for these DEGs with confidence score $>0.4$ [44]. Then, PPI network of DEGs was visualized by Cytoscape [45]. According to the default algorithm of the cytoHubba plugin, ranking nodes indicated the importance of networks [46], which help us locate hub genes. The Molecular Complex Detection (MCODE) plugin was performed to select linked genes with degree cutoff $=2$ and $k$ - core $=2$.

2.6. Construction of a hsa-miR-663b-Related ceRNA Network and the Combination Pathway Analysis of Host Gene. Our research obtained the candidate ceRNA pairs via miRNAtargeted relationship and satisfy the following conditions: (a) the number of identical miRNAs between ceRNA pairs should be greater than 5 and (b) $P$ value $<0.01$ and adjusted FDR $<0.05$. We found that hsa-miR-663b was the key regulator between these selected ceRNA pairs, which was related to many ceRNA pairs. The combination pathway analysis of host gene used the classical algorithm PageRank in the random walk model to obtain the score of all nodes in network. 
Ranging these scores and selecting the top 10 percent as crucial nodes, these nodes were analyzed in pathway enrichment and were mapped into the top 5 enriched pathways. Then, we used igraph $\mathrm{R}$ package to visualize these relationships.

2.7. Western Blot Analysis. 16HBE were lysed in RIPA buffer (Beyotime, P0013), which were grown on 6-well culture plates. Then, equal amounts of extracted proteins (10$30 \mu \mathrm{g}$ ) were separated in 10-15\% SDS-PAGE and transferred to the polyvinylidene difluoride membrane (Immobilon-P Transfer Membranes, IPVH00010, $0.45 \mu \mathrm{m}$ ) by electrophoresis. The membranes were incubated with specific primary antibody for $1 \mathrm{~h}$ at room temperature and afterwards were incubated by anti-mouse IgG, HRP-linked secondary antibody (Cell Signaling Technology, 7076) or anti-rabbit IgG, HRP-linked secondary antibody (Cell Signaling Technology, 7074). When finished the above footsteps, the membranes were detected by chemiluminescence detection (Tannon 4800). The grayscale of the bands was quantified using ImageJ (Version 1.58j8), and the data were normalized to the GAPDH loading controls.

2.8. Electron Microscopy (EM). $16 \mathrm{HBE}$ were stimulated with different concentrations of LPS for $12 \mathrm{~h}$. After treatment, $16 \mathrm{HBE}$ were fixed with $2 \%$ glutaraldehyde/0.1 M phosphate buffer ( $\mathrm{pH} 7.4$ ) and in $1 \%$ osmium tetroxide/0.1 M phosphate buffer ( $\mathrm{pH}$ 7.4) and dehydrated with a graded series of ethanol. Then, we embedded these fixed 16HBE into epoxy resin. Ultrathin sections were stained with lead citrate and uranyl acetate. The sections were observed with the Hitachi H-7500 transmission electron microscope (Hitachi, Tokyo, Japan). For quantitative evaluation of autophagosomes in 16HBE, 16 image fields ( 8 of $1500 \mathrm{x}$ and $5000 \mathrm{x}$, respectively) were selected for each sample.

2.9. Immunofluorescence Staining. $16 \mathrm{HBE}$ cells grown on 36well culture slides were fixed with $4 \%$ paraformaldehyde for $15 \mathrm{~min}$ and were permeated with $0.03 \%$ Triton X-100 (Solarbio, T8200) for $20 \mathrm{~min}$. 5\% BSA (BioFroxx, 4240) was used to block cells for $30 \mathrm{~min}$; then, the primary antibodies were incubated for 12-18 hours and secondary antibodies were incubated for 1 hour at room temperature. Fluorescence microscopy (Olympus, IX81, Japan) analysis of LC3B and DAPI staining were performed in $16 \mathrm{HBE}$.

2.10. Statistical Analysis. Statistical analysis was performed using GraphPad Prism 7.00 and R 4.0.5 software. Date are shown as the average ( \pm SEM) taken from at least 3 independent experiments. Parametric data between 2 different groups were compared by the Student $t$-test. The variance for multiple comparisons was determined by one-way analysis. Significance was defined as $P<0.05$. The statistical methods of bioinformatics analysis were detailed as mentioned previously.

\section{Results}

3.1. LPS-Induced Autophagy in Lung Epithelial Cells. To explore the alteration of autophagy when 16HBE suffered from the stimulation of LPS, we set different concentrations of LPS and observed the changes of autophagy via western blotting, immunofluorescence staining, and electron microscopy (Figure 1). To preliminarily confirm the variation trend of autophagy in LPS-induced 16HBE cell lines, we detected the expression of microtubule-associated protein 1 light chain 3 beta (MAP1LC3B) and sequestosome 1 (SQSTM1/p62). The expression of MAP1LC3B-I and SQSTM1/p62 appeared similar trend which rose firstly along with the higher concentration of LPS and reached climax in $1000 \mathrm{ng} / \mathrm{ml}$ of LPS. Nevertheless, the expression of them declined with unceasingly increasing concentration of LPS. The expression of MAP1LC3B-II was apparently upregulated compared to the negative control group, whereas it showed no obvious discrepancy between the LPS-induced groups (Figure 1(a)). In immunofluorescence, we labeled autophagosomes and nuclei with LC3-II punctate dots and DAPI which emitted red and blue colors, respectively (Figure 1(b)). We set the NC group and RAPA group as the negative and positive control groups and found that there were more LC3B punctate dots in the $1000 \mathrm{ng} / \mathrm{ml}$ LPS-stimulated group. Subsequently, we verified the identical phenomenon through electron microscopy (EM) evaluation (Figure 1(c)). LPS significantly increased the number of autophagic vacuoles, and the change was mostly obvious in the $1000 \mathrm{ng} / \mathrm{ml}$ LPSstimulated group (Figure 1(d)). Under observations, most vacuoles were late/degradative autophagic vacuoles/autolysosomes (AVd or $\mathrm{AVl}$ ) in our research which typically had only one limiting membrane and contained electrondense cytoplasmic material.

3.2. Differentially Expressed Genes in LPS-Stimulated Lung Epithelial Cells. We constructed 8 strains of 16HBE into two groups as the negative control group and LPSstimulated group, and they all included 4 strains of the isogenous 16HBE. The stimulative concentration of LPS was $1000 \mathrm{ng} / \mathrm{ml}$, and stimulative time was 12 hours. Then, we made the whole transcriptome sequencing analysis in these cells to figure out the alterations in 16HBE after LPSrelated stimulation. We obtained 750 DEGs and constructed volcano plot to display the distribution of DEGs in the dimensions of $-\log _{10}(P$ value $)$ and $\log _{2}(\mathrm{FC})$. On the basis of $P<0.05$ and $|\mathrm{FC}|>1.5,312$ genes were upregulated and 438 were downregulated as shown in Figure 2(a). To intuitively understand the overall distribution of expression levels and fold changes, we also demonstrate the results in MA plot (Figure 2(b)). We made hierarchical clustering analysis and display it in heat map (Figure 2(c)).

3.3. Differentially Expressed Noncoding RNAs in LPSStimulated Epithelial Cells. Except for sequencing in mRNAs, we also sequenced noncoding RNAs (ncRNA) including lncRNAs, circRNAs, and miRNAs. The volcano plots and heat maps of differentially expressed noncoding RNAs are shown in Figures 3(a)-3(f). Similarly, we set the screen criteria as $P<0.05$ and $|\mathrm{FC}|>1.5$. We found more differentially expressed ones in lncRNAs, which contained 230 upregulated and 219 downregulated lncRNA. circRNAs only revealed 76 differentially expressed ones in our study 

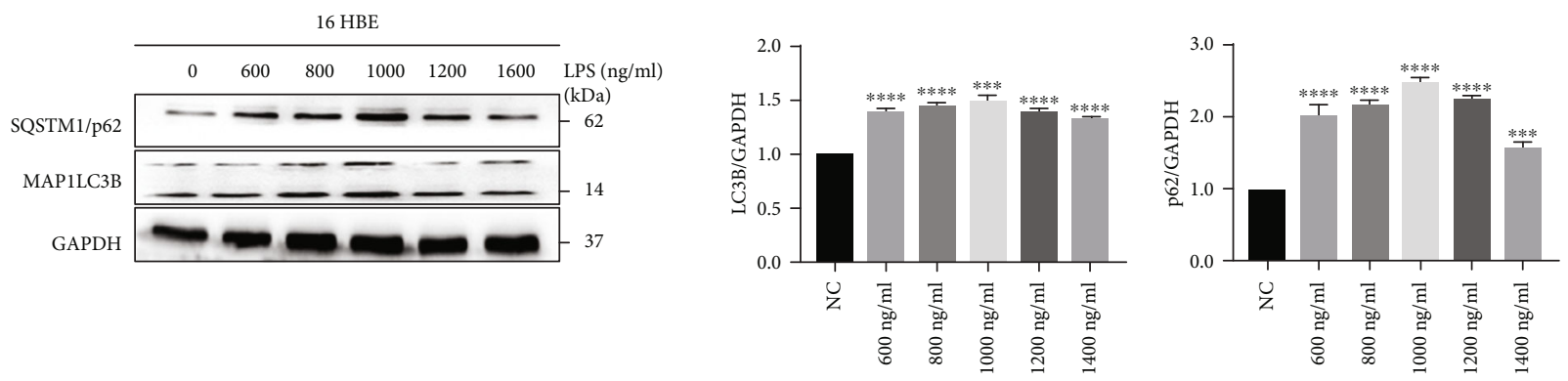

(a)

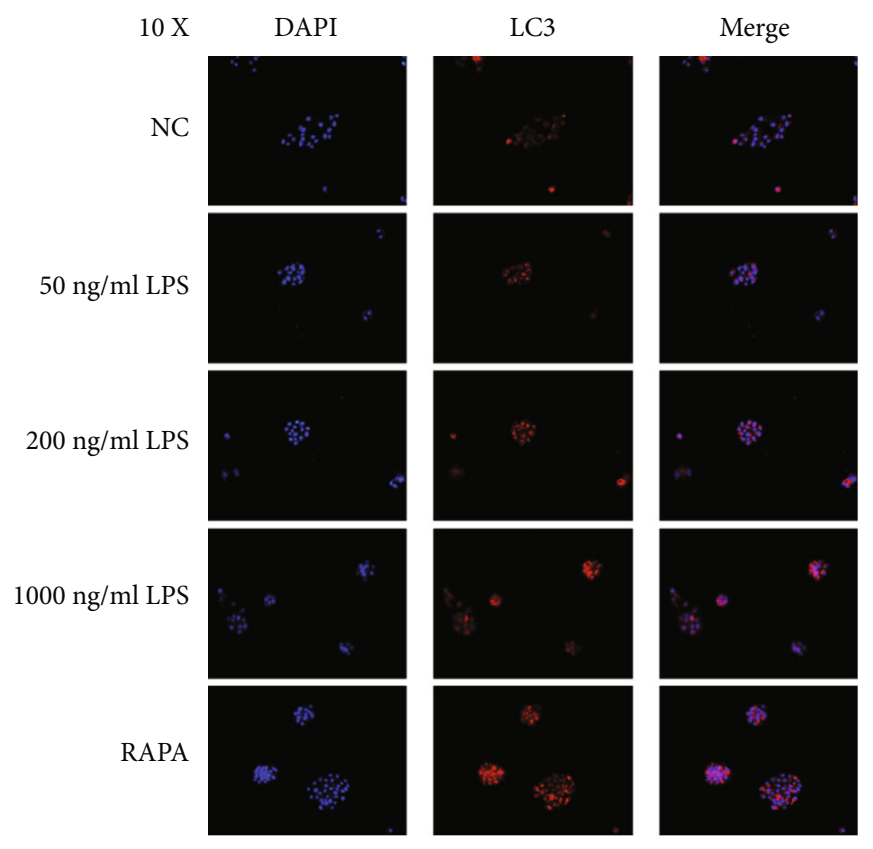

(b)

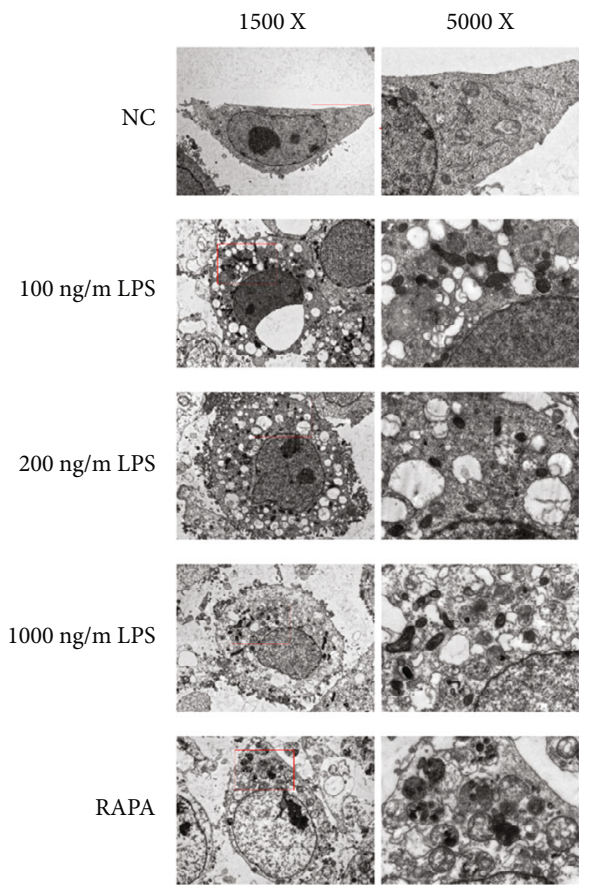

(c)

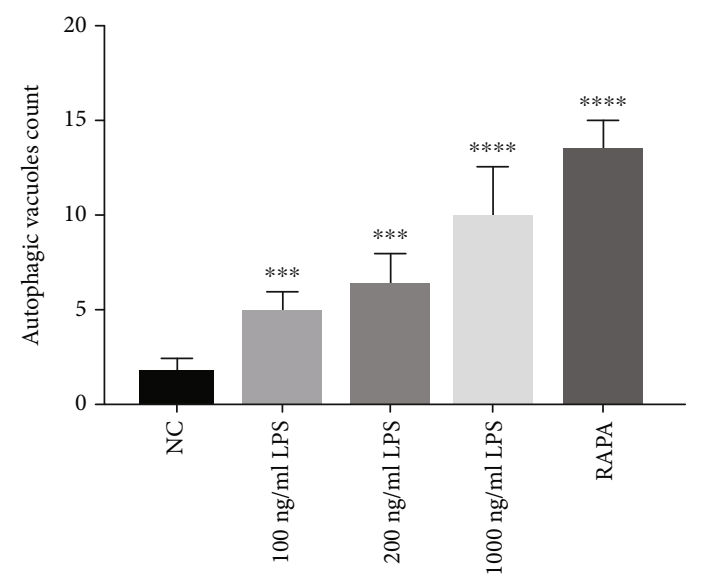

(d)

FIGURE 1: LPS induces autophagy in 16HBE. (a) WB using anti-MAP1LC3B, anti-SQSTM1/p62, and anti-GAPDH. 16HBE were treated with different concentrations of LPS $(0-1600 \mathrm{ng} / \mathrm{ml})$ and RAPA $(50 \mathrm{nM})$ for $12 \mathrm{~h}$, and protein samples were collected. Shown is a representative experiment of 2 showing similar results. The middle panel is the average $( \pm \mathrm{SEM})$ of the relative increase in LC3B normalized to GAPDH and the right panel is the average $( \pm$ SEM) of the relative increase in SQSTM1/p62 normalized to GAPDH. ${ }^{* * *} P<0.001$ and ${ }^{* * * *} P<0.0001$. (b) Fluorescence microscopy detection (10x) of DAPI (left panels) and LC3 (right panels). 16HBE were treated with different concentrations of LPS $(0-1600 \mathrm{ng} / \mathrm{ml})$ for $12 \mathrm{~h}$. (c) Electron microscopy detection of autophagosome in $16 \mathrm{HBE}$ with different concentrations of LPS $(0-1600 \mathrm{ng} / \mathrm{ml})$ and RAPA $(50 \mathrm{nM})$. (d) Shown in this panel is average $( \pm$ SEM) of autophagosome counts taken from 6 image fields $(1500 \mathrm{x})$ for each sample. ${ }^{* * *} P<0.001$ and ${ }^{* * * *} P<0.0001$. 


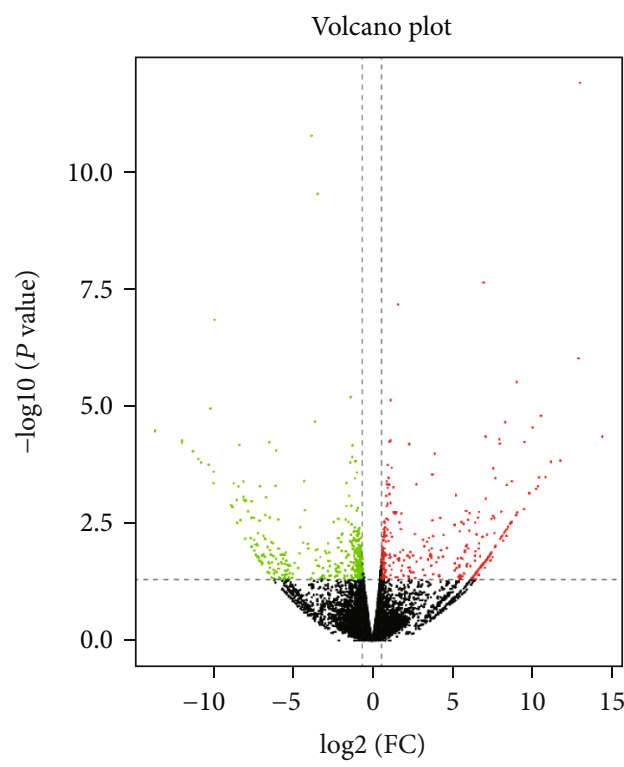

Significant

$\square$ Up

$\square$ Down

$\square$ Normal

(a)

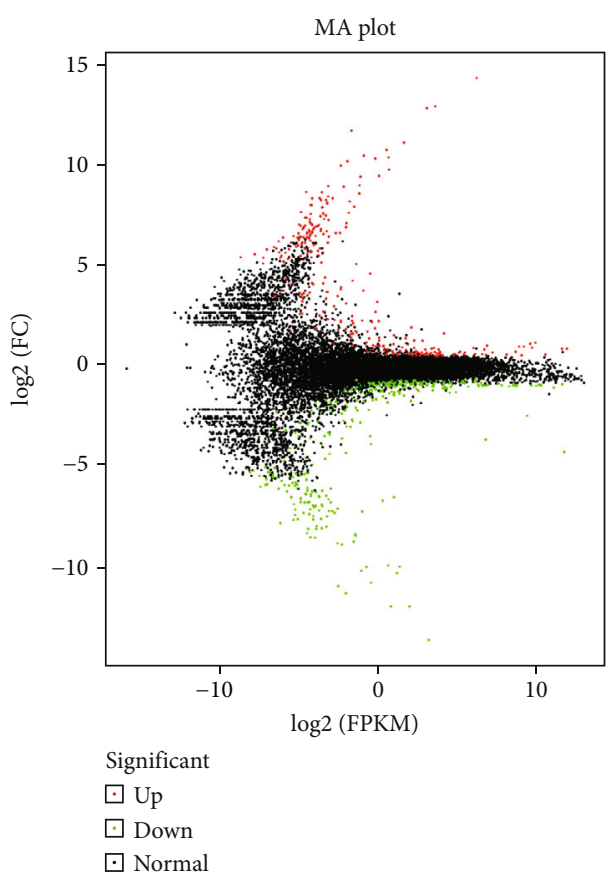

(b)

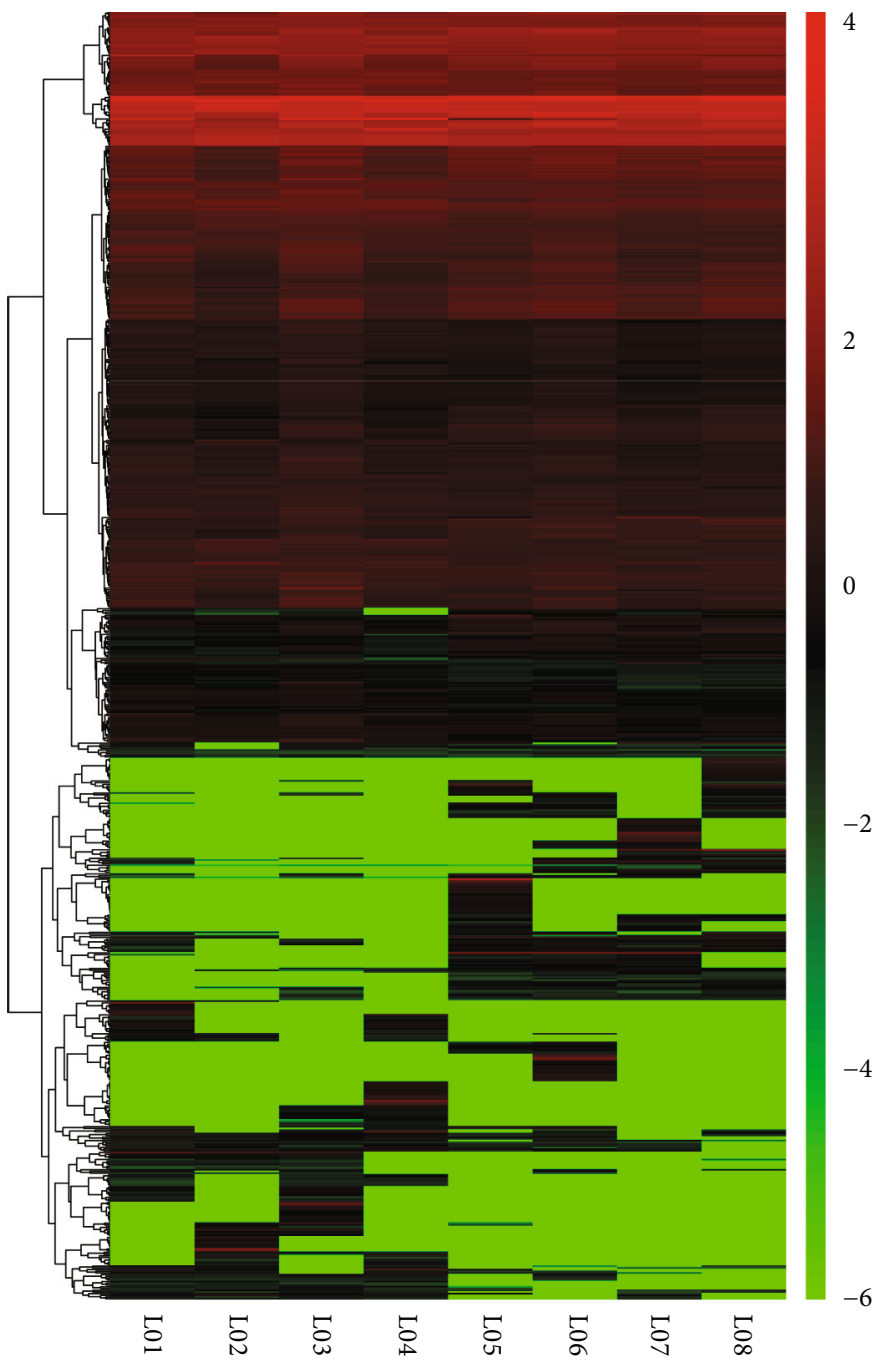

(c)

Figure 2: Comprehensive analysis of differentially expressed genes. (a) The volcano plot of differentially expressed genes. (b) The MA plot of differentially expressed genes. (c) The heat map of differentially expressed genes.

(Table 1). These heat maps demonstrated that there were existing variations between the negative control groups and LPS-stimulated groups which furtherly prompted underlying ceRNA regulatory network in $16 \mathrm{HBE}$ when suffering exposure of LPS (Figure 3).
3.4. Enrichment Analysis of Differentially Expressed Genes and ncRNAs in LPS-Stimulated Epithelial Cells. To investigate functions of the differentially expressed genes and ncRNAs, we performed GO and KEGG using clusterProfiler of R. Enriched terms with $P<0.05$ were displayed (Figure 4 


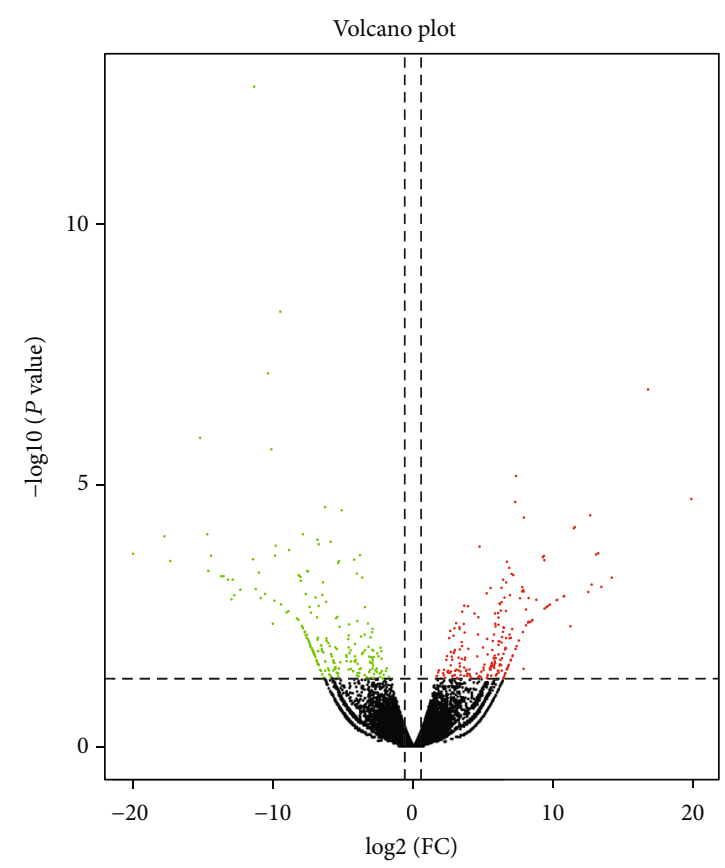

Significant

$\square$ Up

$\square$ Down

$\square$ Normal

(a)

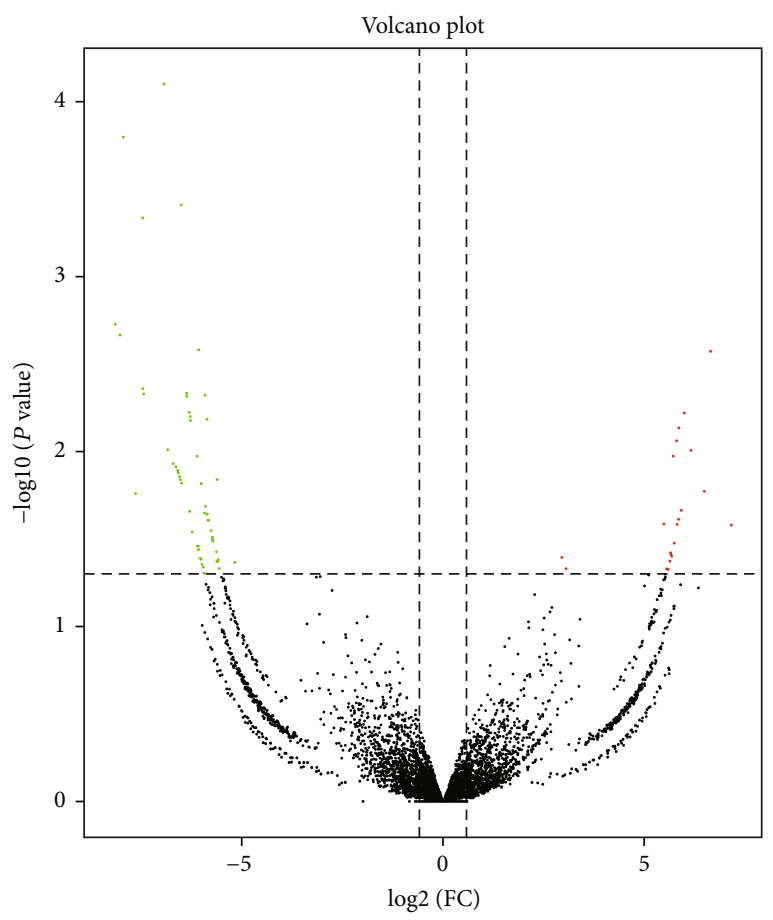

Significant

$\square$ Up

$\square$ Down

$\square$ Normal

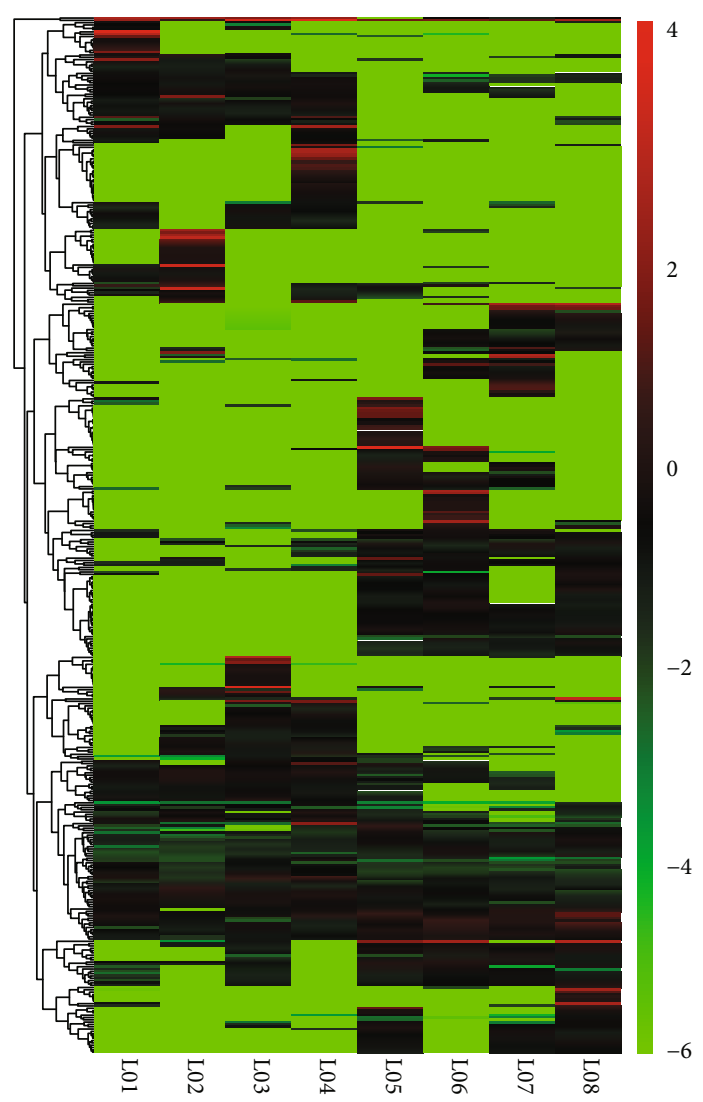

(b)

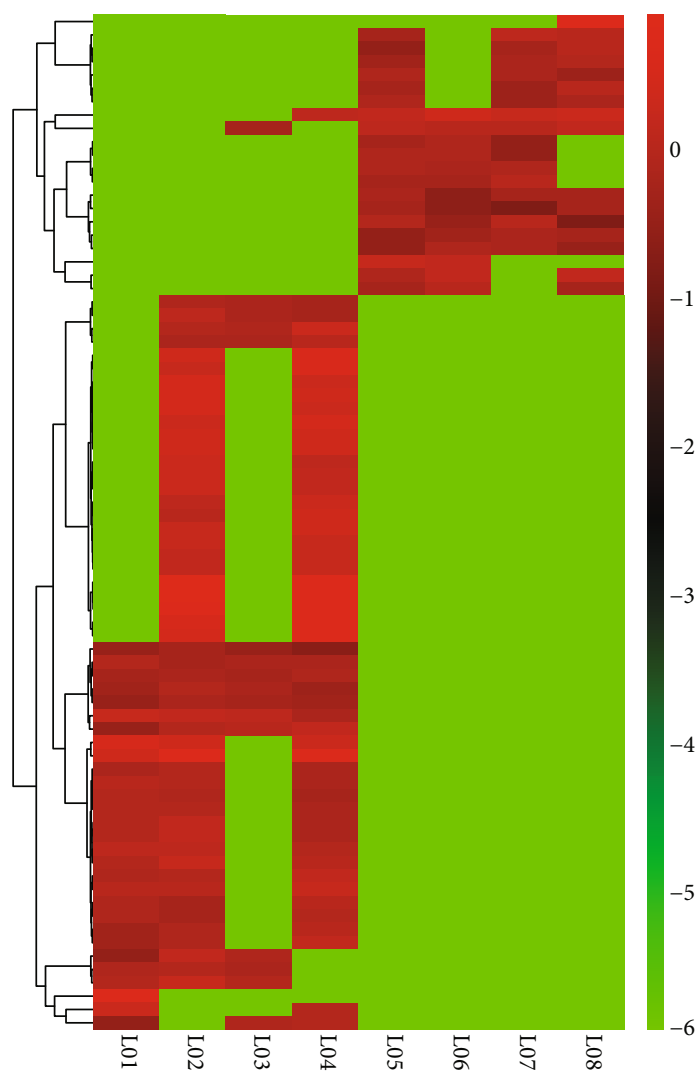

(d)

(c)

Figure 3: Continued. 


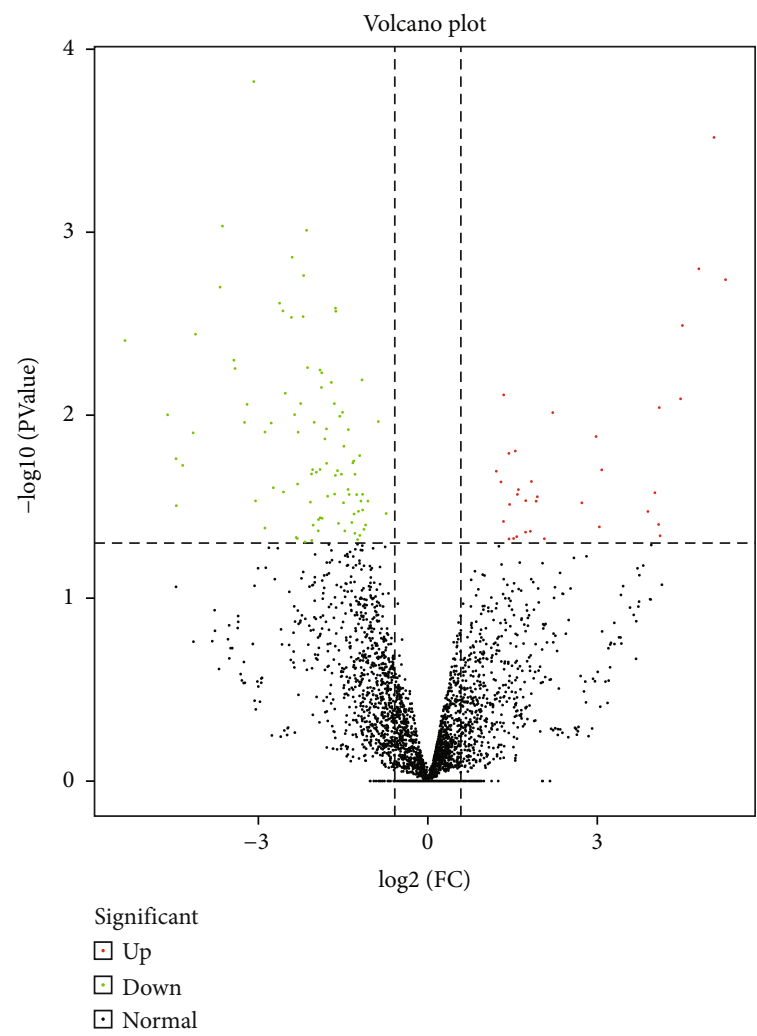

(e)

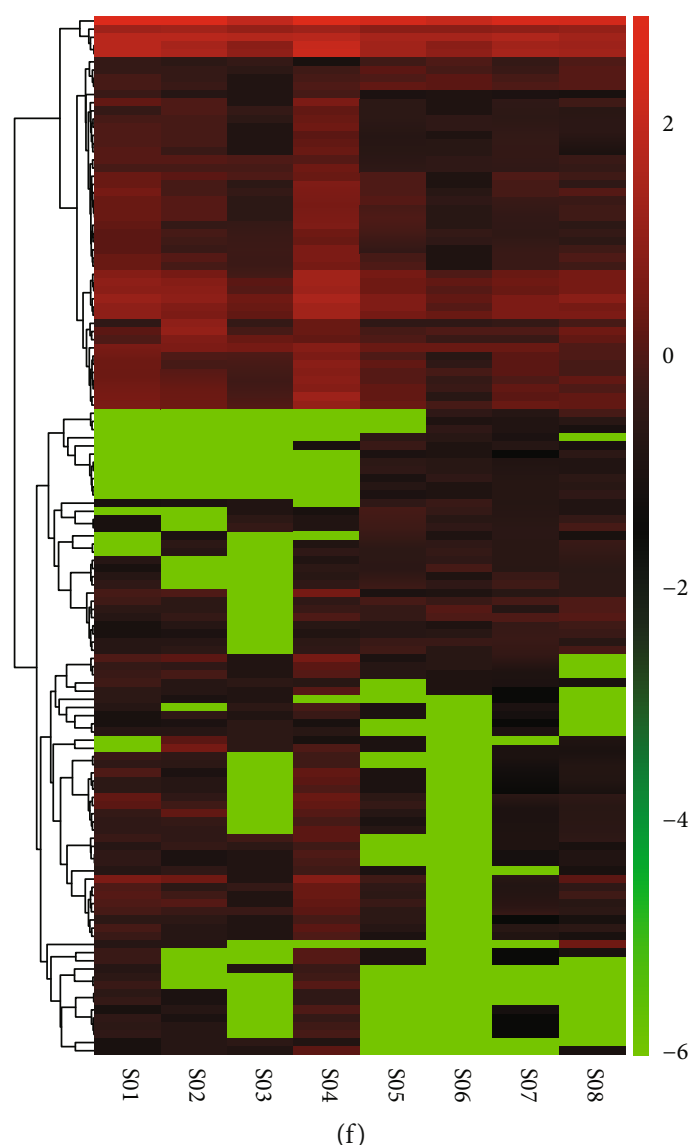

(f)

Figure 3: Comprehensive analysis of differentially expressed RNAs. (a) The volcano plot of differentially expressed lncRNAs. (b) The heat map of differentially expressed lncRNAs. (c) The volcano plot of differentially expressed circRNAs. (d) The heat map of differentially expressed circRNAs. (e) The volcano plot of differentially expressed miRNAs. (f) The heat map of differentially expressed miRNAs.

TABLE 1: The differentially expressed RNAs in LPS-stimulated 16HBE.

\begin{tabular}{lccc}
\hline $\begin{array}{l}\text { DEG set } \\
\text { (L01_L02_L03_L04 } \\
\text { vs. L05_L06_L07_L08) }\end{array}$ & All DEG & Upregulated & Downregulated \\
\hline lncRNA & 449 & 230 & 219 \\
circRNA & 76 & 21 & 55 \\
miRNA & 127 & 34 & 93 \\
\hline
\end{tabular}

The L01 represents the first strain of $16 \mathrm{HBE}$, and the rest can be known in the same way.

and S1). The genes were mainly enriched in viral transcription, nuclear-transcribed mRNA catabolic process, and SRP-dependent cotranslational protein targeting to the membrane in biological process; CD40 receptor complex and cytoplasmic side of the plasma membrane in cellular component; structural constituent of ribosome and mitogen-activated protein kinase (MAPK) kinase kinase binding in molecular function (Figures 4(a) and 4(b)); and ribosome, Parkinson's disease, and oxidative phosphorylation in KEGG (Figure 4(c)). The cis-targeted genes of lncRNAs were enriched in blood coagulation and Fcepsilon receptor signaling pathway in biological process
(Figure S1A), the lysosomal membrane and endosome membrane in cellular component (Figure S1B), identical protein binding and histone deacetylase binding in molecular function (Figure S1C), and HTLV-I infection and Herpes simplex infection in KEGG (Figure S1D). The trans-targeted genes of lncRNAs were enriched in nucleartranscribed mRNA catabolic process and translation initiation in biological process (Figure S1E), CD40 receptor complex in cellular component (Figure S1F), structural constituent of ribosome and ubiquitin-conjugating enzyme binding in molecular function (Figure S1G), and ribosome, oxidative phosphorylation, and Parkinson's disease in KEGG (Figure S1H). The circRNAs were mainly enriched in positive regulation of cell migration in biological process (Figure S1I), while there was no predominant result in KEGG under limitation of $P<0.05$. The miRNA was mainly enriched in dendrite and neuron projection in cellular component (Figure S1J), protein kinase binding in molecular function (Figure S1K), and pathway in cancer, endocytosis, and the MAPK signaling pathway in KEGG (Figure S1L).

3.5. Construction of the PPI Network and Screening of Modules and Hub Genes. To furtherly figure out the significant proteins and biological modules which played a vital role in the LPS-induced ALI, we constructed PPI network 


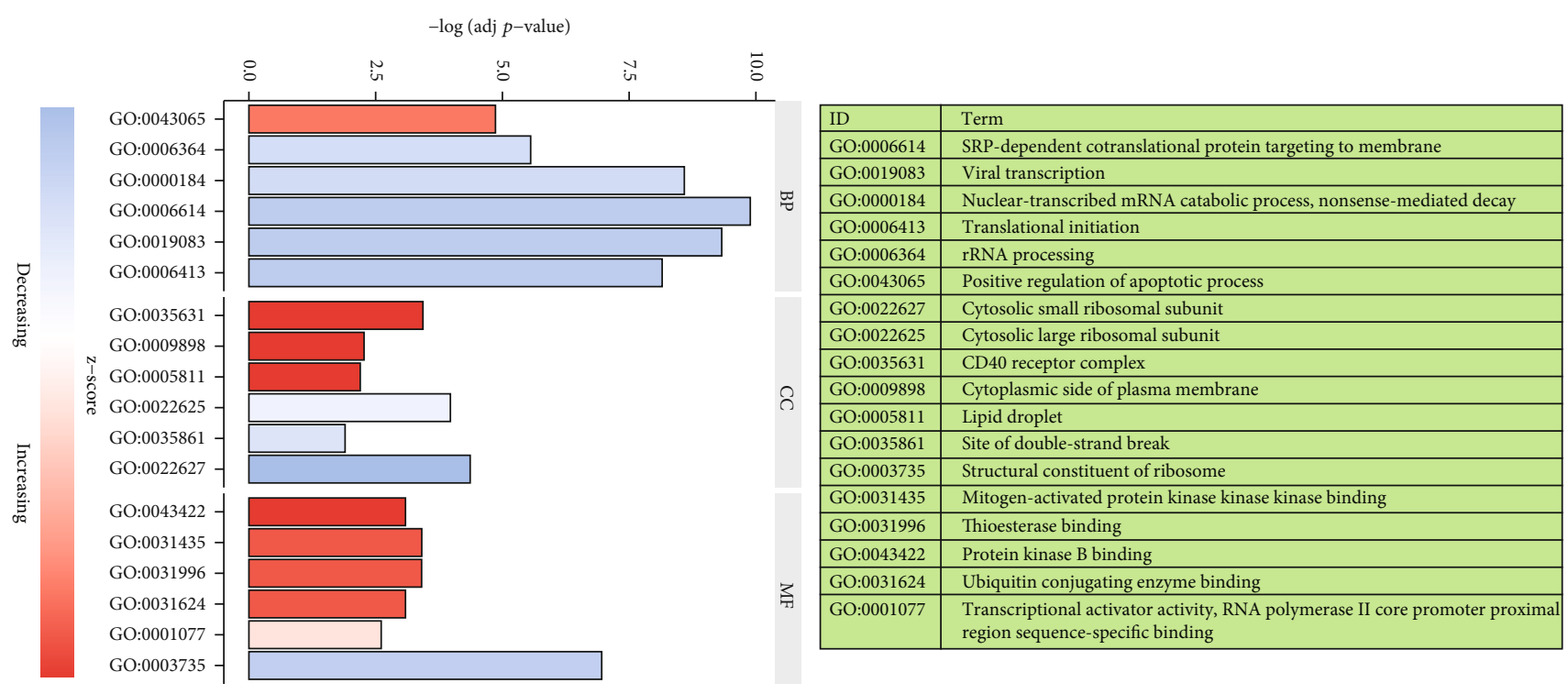

(a)

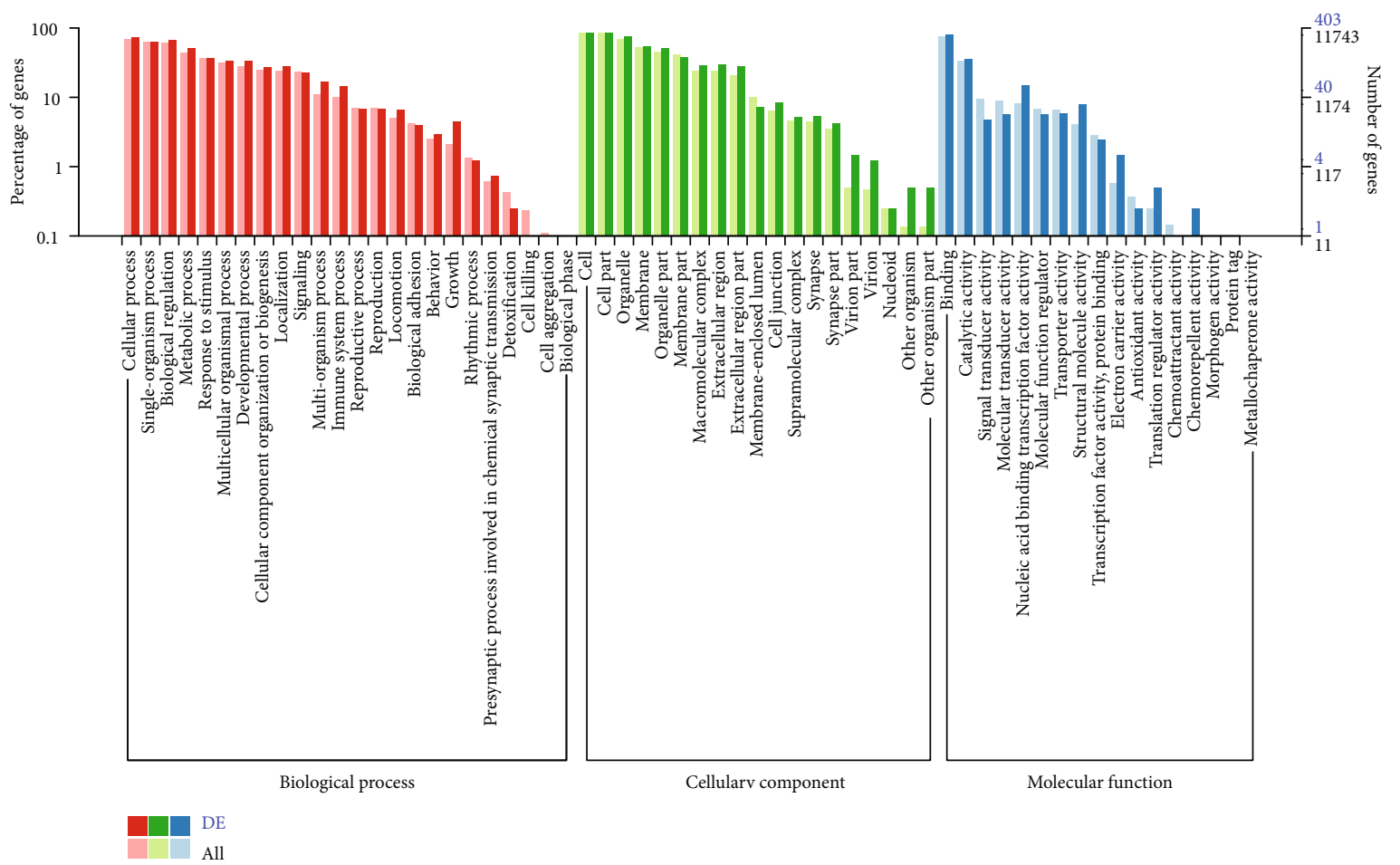

(b)

FIgUre 4: Continued. 


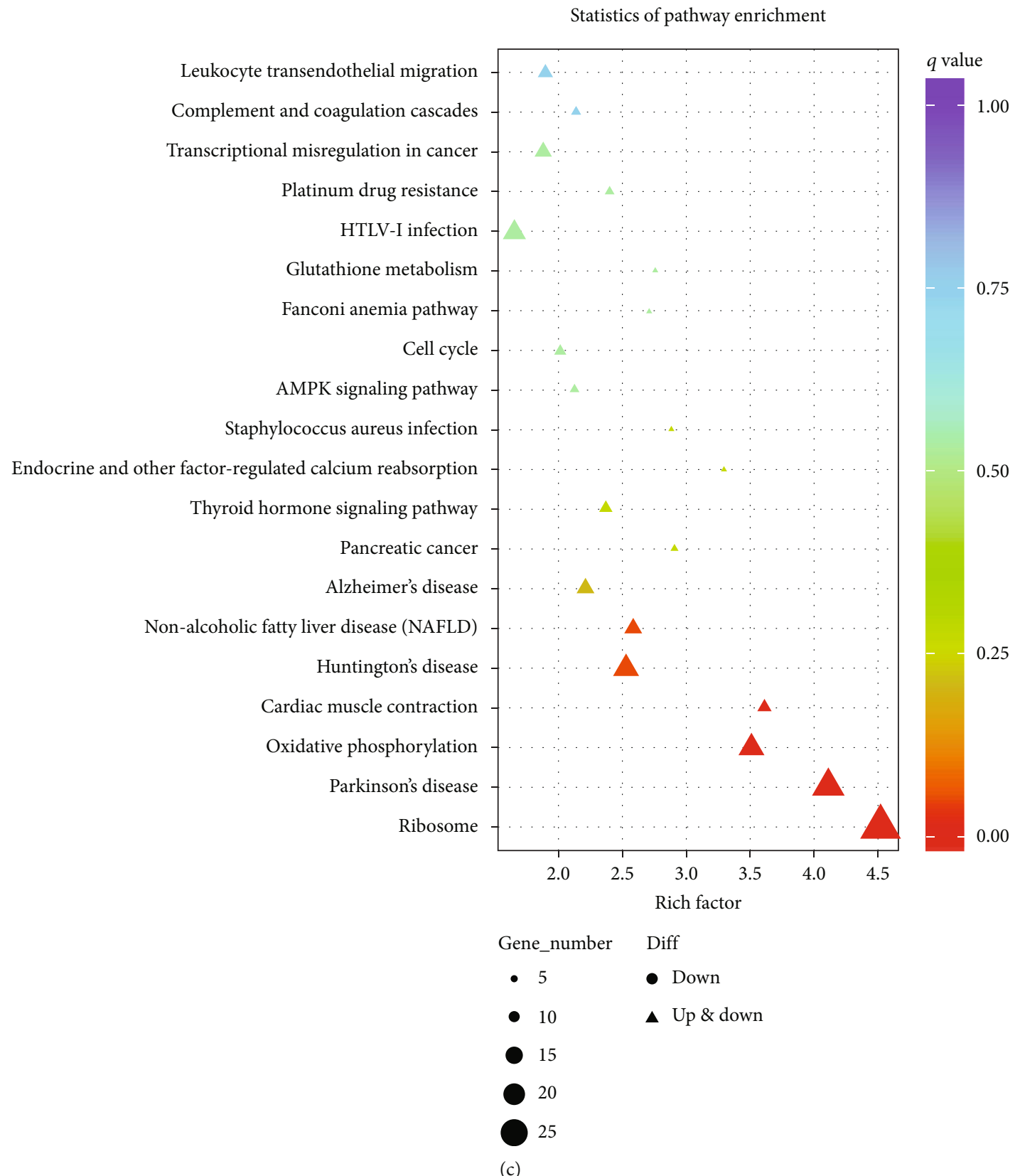

FIGURE 4: Functional enrichment analysis of differentially expressed genes in LPS-stimulated 16HBE. (a) Gene Ontology terms of differentially expressed genes in LPS-stimulated 16HBE. The right panel is the annotations of ID of GO terms. (b) The discrepancy of GO terms between differentially expressed genes and whole genes. (c) The KEGG enrichment analysis of differentially expressed genes in LPS-stimulated $16 \mathrm{HBE}$ via clusterProfiler R package. The horizontal ordinate is enrichment factor which shows the ratio of differentially expressed genes enriched in the pathway to whole genes enriched in the pathway. The vertical ordinate is $-\log _{10}\left(Q-v^{-v a l u e}\right)$.

via utilizing STRING and visualized the differentially expressed genes through Cytoscape software and cytoHubba (Figure 5(a)). Then, we located top 12 hub genes (UPF2, MYC, RPL34, RPL39, RPS3A, RPS23, RPS7, RPL7, RPL31, RPS24, RPS27A, RPS10-NUDT3) by calculating maximal clique centrality (MCC) which was the top 1 module and revealed the most densely connected region (Figure 5(b)). We selected 36 genes (IL6, RPS27A, MYC, ESR1, CCND1, CDC42, COX7C, RPS3A, ITGAM, PSMA6, FBXW7,
DYNC112, TXN, MT-CYB, RPS23, MT-CO2, RAD51, SOCS3, HINT1, RPS7, RPL34, RPS24, KCNQ1, TOP2A, NDUFA5, MT-CO3, B2M, RAN, CKAP5, HIST1H2AD, SMC3, BCL2L1, RAB11A, RPL31, CXCL1, CP) via degree analysis (degree value $\geq 15$ ) and made functional annotation of these 36 genes by $\mathrm{M}$-code which illustrated the vital functions in $6 \mathrm{GO}$ terms $(P<0.05)$. The results are showed in Table 2 and contained RNA binding, nuclear-transcribed mRNA catabolic process, nonsense-mediated decay, poly(A) 


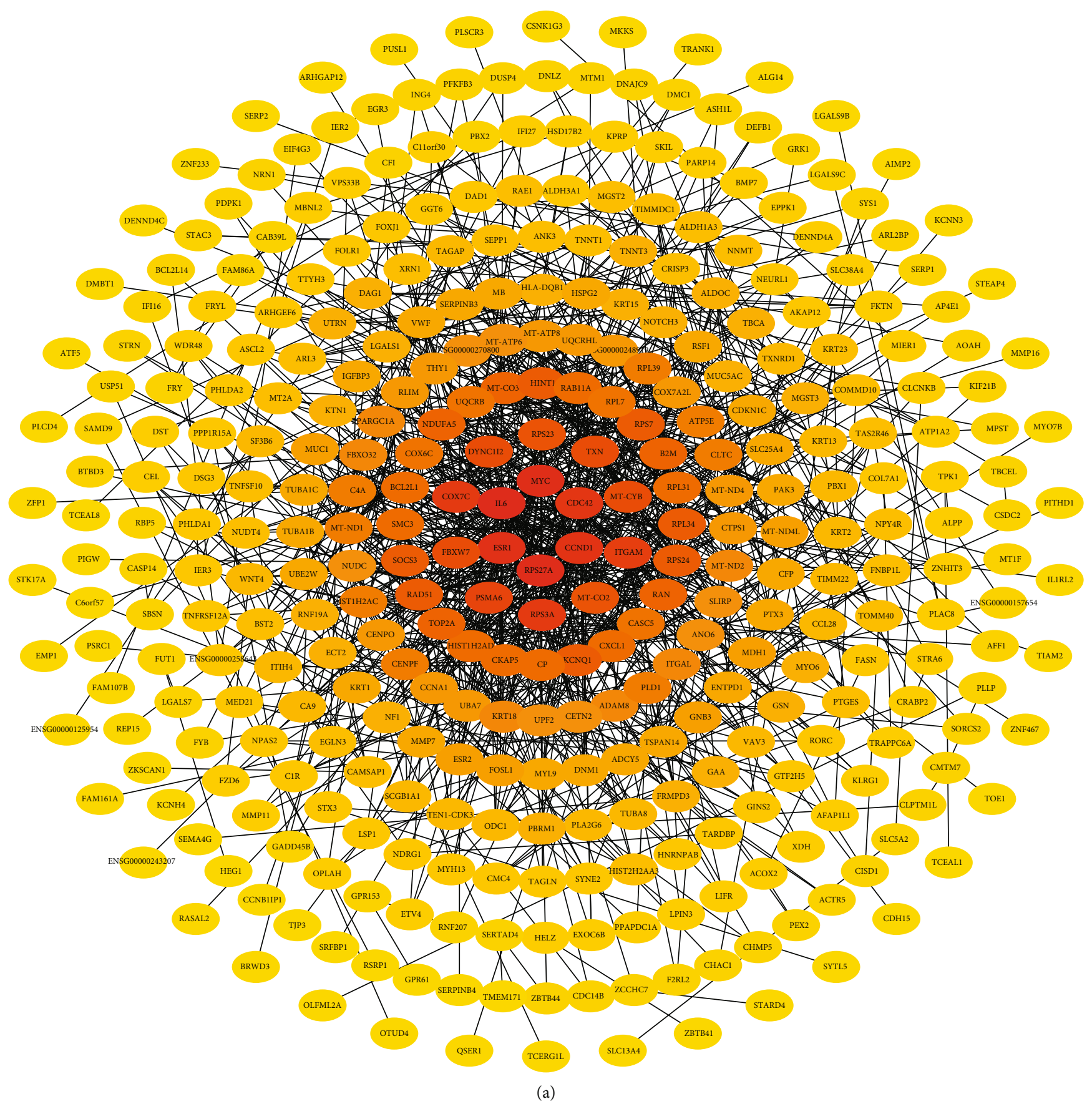

Figure 5: Continued. 


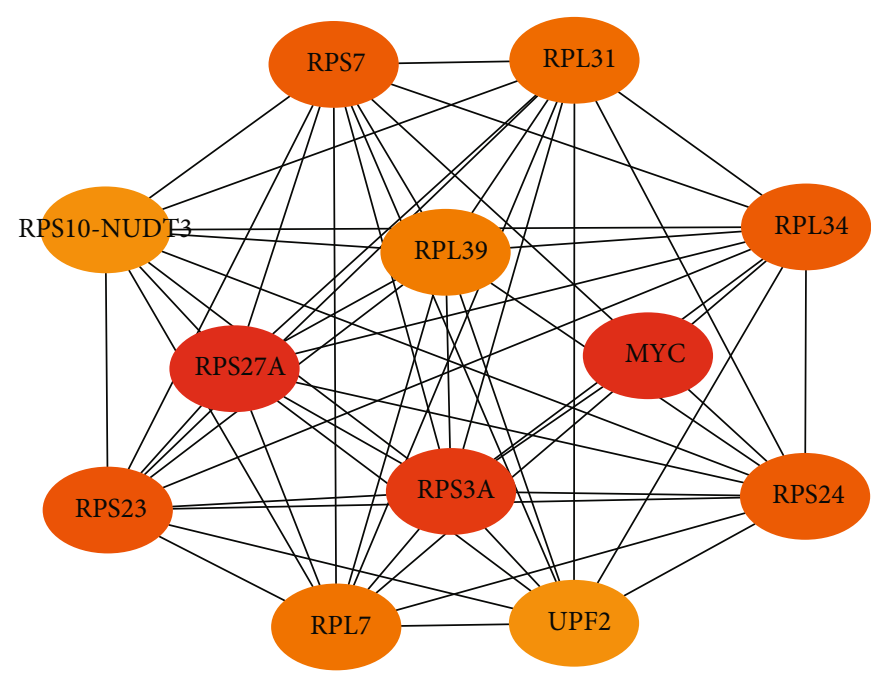

(b)

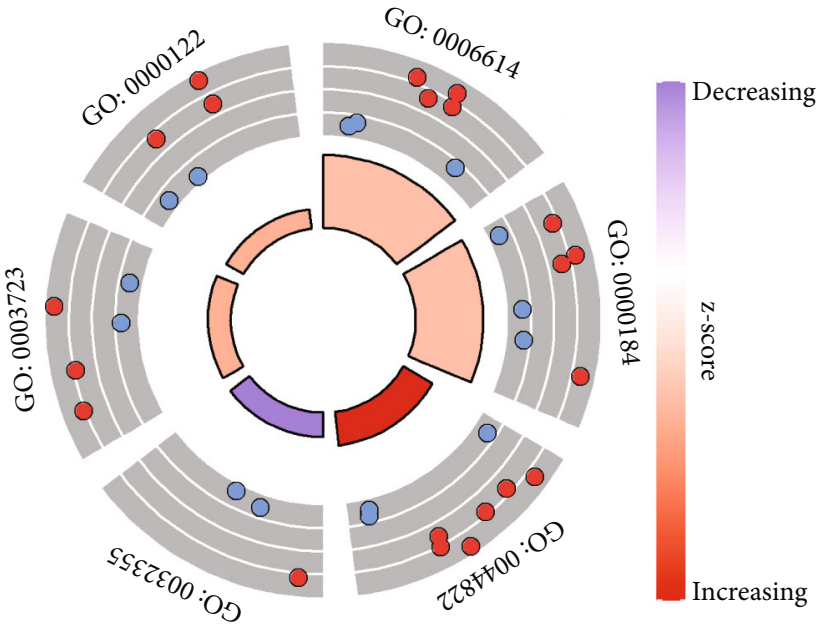

$\log \mathrm{FC}$

Downregulated

- Upregulated

(c)

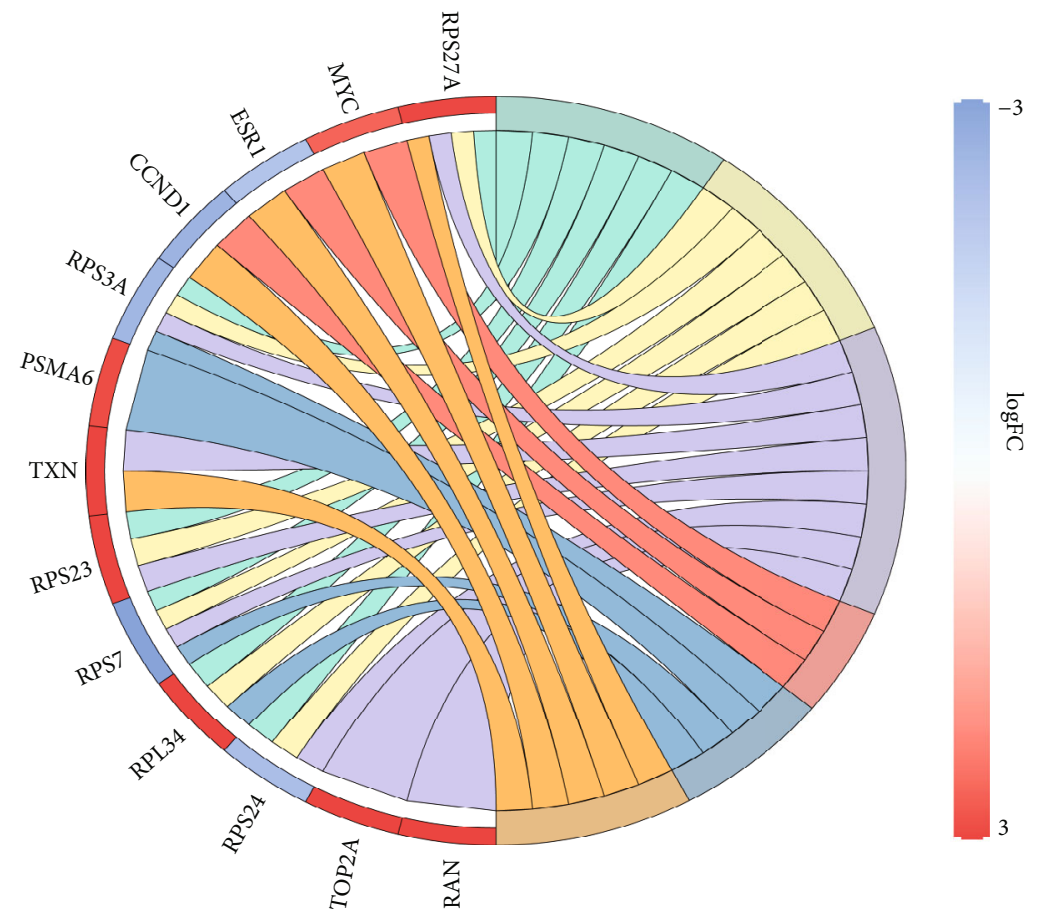

GO Terms
SRP-dependent contranslational protein targeting to membrane
Nuclear-transcribed mRNA catabolic process, nonsense-mediated decay
Negative regulation to transcription from RNA polymerase II promoter
Poly(A) RNA binding
Response to estradiol
RNA binding

(d)

FIGURE 5: Screening the key genes and further GO analysis from PPI network. (a) The PPI network constructed via STRING. (b) Hub genes selected using cytoHubba. (c) The outer circle represents the expression (logFC) of 36 differentially expressed genes in each enriched GO (Gene Ontology) terms. The inner circle indicates the significance of GO terms $\left(\log _{10}\right.$-adjusted $P$ values). Red points indicate upregulated gene, and blue points indicate downregulated gene. (d) The circle indicates the correlation between hub genes and their Gene Ontology terms. 
TABLE 2: The top 6 GO terms of 36 differentially expressed genes.

\begin{tabular}{|c|c|c|c|c|}
\hline Category & ID & Term & Genes & Adj_Pval \\
\hline $\mathrm{BP}$ & GO:0006614 & $\begin{array}{l}\text { SRP-dependent cotranslational protein } \\
\text { targeting to membrane }\end{array}$ & RPS7, RPL31, RPL34, RPS3A, RPS27A, RPS24, RPS23 & $2.11 E-08$ \\
\hline $\mathrm{BP}$ & GO:0000184 & $\begin{array}{l}\text { Nuclear-transcribed mRNA catabolic } \\
\text { process, nonsense-mediated decay }\end{array}$ & RPS7, RPL31, RPL34, RPS3A, RPS27A, RPS24, RPS23 & $8.70 E-08$ \\
\hline MF & GO:0044822 & Poly(A) RNA binding & $\begin{array}{c}\text { TOP2A, RPS7, RPL31, RPS3A, TXN, RPS27A, } \\
\text { RPS24, RAN, RPS23 }\end{array}$ & $9.68 E-04$ \\
\hline $\mathrm{BP}$ & GO:0032355 & Response to estradiol & CCND1, MYC, ESR1 & 0.012963812 \\
\hline MF & GO:0003723 & RNA binding & PSMA6, RPS7, RPL31, RPL34, RPS3A & 0.019110457 \\
\hline $\mathrm{BP}$ & GO:0000122 & $\begin{array}{l}\text { Negative regulation of transcription } \\
\text { from RNA polymerase II promoter }\end{array}$ & CCND1, MYC, TXN, RPS27A, ESR1 & 0.046553861 \\
\hline
\end{tabular}

BP: biological process; MF: molecular function.

RNA binding, response to estradiol, SRP-dependent cotranslational protein targeting to the membrane, and negative regulation of transcription from RNA polymerase II promoter (Figures 5(c) and 5(d)).

3.6. Combined Analysis and Construction of hsa-miR-663bRelated ceRNA Network. The differential analysis of whole transcriptome showed differentially expressed mRNA, miRNA, IncRNA, and circRNA of multiple groups, and the overall variation significance were exhibited through Circos (Figure 6(a)). The observation of variations of multiomics data in whole transcriptome was attributed to discover the differential regulatory mechanism. We used the classical algorithm PageRank in the random walk model to obtain the score of all nodes in network which represented the importance in network. We made pathway enrichment analysis of the crucial RNAs in network and then selected the first five pathways with most significant enrichment to illustrate the gene interactions of these pathways via igraph $R$ package (Figure 6(b)). To better predict the upstream regulatory mechanism in the progression of LPS-induced ALI, we constructed a ceRNA network to elaborate the regulatory relationships. According to the whole transcriptome analysis, we took the DElncRNAs or DEcircRNAs as the center and matched corresponding targeted relationships with DEGs and DEmiRNAs to constitute DElncRNADEmiRNA-DEG ceRNA pairs (Table 3) and there were no DEcircRNA-DEmiRNA-DEG ceRNA pairs by screening. In these differentially expressed RNA, hsa-miR-663b exerted a central regulatory function (Figure 6(c)). Hence, we presented a hsa-miR-663b-related ceRNA network to explain the regulatory mechanism in LPS-induced ALI, which was conductive for us to furtherly explore the diagnosis and intervention methods of ALI.

\section{Discussion}

ALI was a form of parenchymal lung disease which had various etiologies and usually leaded to fulminant respiratory failure and death. It could develop to acute respiratory distress syndrome (ARDS) and had approximately 33\% mortality rate leading to consumption of significant healthcare resources globally [47]. In recent years, it had obtained some achievements in a better comprehension of ALI pathophysiology and proposed some novel measures to pharmacotherapy such as corticosteroid, N-acetylcysteine, statins, surfactants, and antibiotics. Nevertheless, these therapies still had not obviously declined the mortality and morbidity of ALI patient, especially the critical ones [48]. The central feature of ALI was an excessive inflammatory response, but autophagy, as a process for maintaining cellular homeostasis, exerted an ambiguous effect in ALI [8, 49-51]. The activation of mTOR facilitated LPSinduced ALI in mouse, and similarly, 3-MA increased the cytotoxicity of A549 alveolar epithelial cells [8, 49]. The above results proved that inhibition of autophagy generated harmful influence and demonstrated that autophagy plays a protective role in pathogenesis of ALI. While some researchers proposed the opposite view, they discovered that the mechanical ventilation may activate nucleotidebinding oligomerization domain-like receptor containing pyrin domain 3 (NLRP3) inflammasome, which could be suppressed by silencing ATG-5 [50]. Song et al. thought that excessive activating autophagy of alveolar type II epithelial (AT-II) cells is a major cause of ALI and found that microRNA-34a could inhibit the excessive activation of autophagy to relieve LPS-induced ALI via targeting forkhead box O3 (FOXO3) [51]. To investigate the autophagy-related regulatory mechanism in ALI, we constructed LPS-induced ALI model in 16HBE human bronchial epithelial cells.

To observe the alterations of autophagy in 16HBE under LPS exposure, we utilized western blot, immunofluorescent staining, and electron microscopy to evaluate changes of autophagy-related protein expression levels and autophagic vacuole amounts. The expressions of LC3B-I and p62 were obviously upregulated under exposure of LPS and reached the highest levels with the LPS concentration of $1000 \mathrm{ng} / \mathrm{ml}$. Although the expression of LC3B-II was likewise increased compared to the negative control group, it did not appear a significant difference $(P$ value $>0.05)$ under the stimulation of different concentrations of LPS. LC3 were primary Atg8-family homolog examined in mammalian cells and the classic autophagosome marker in autophagy-related 


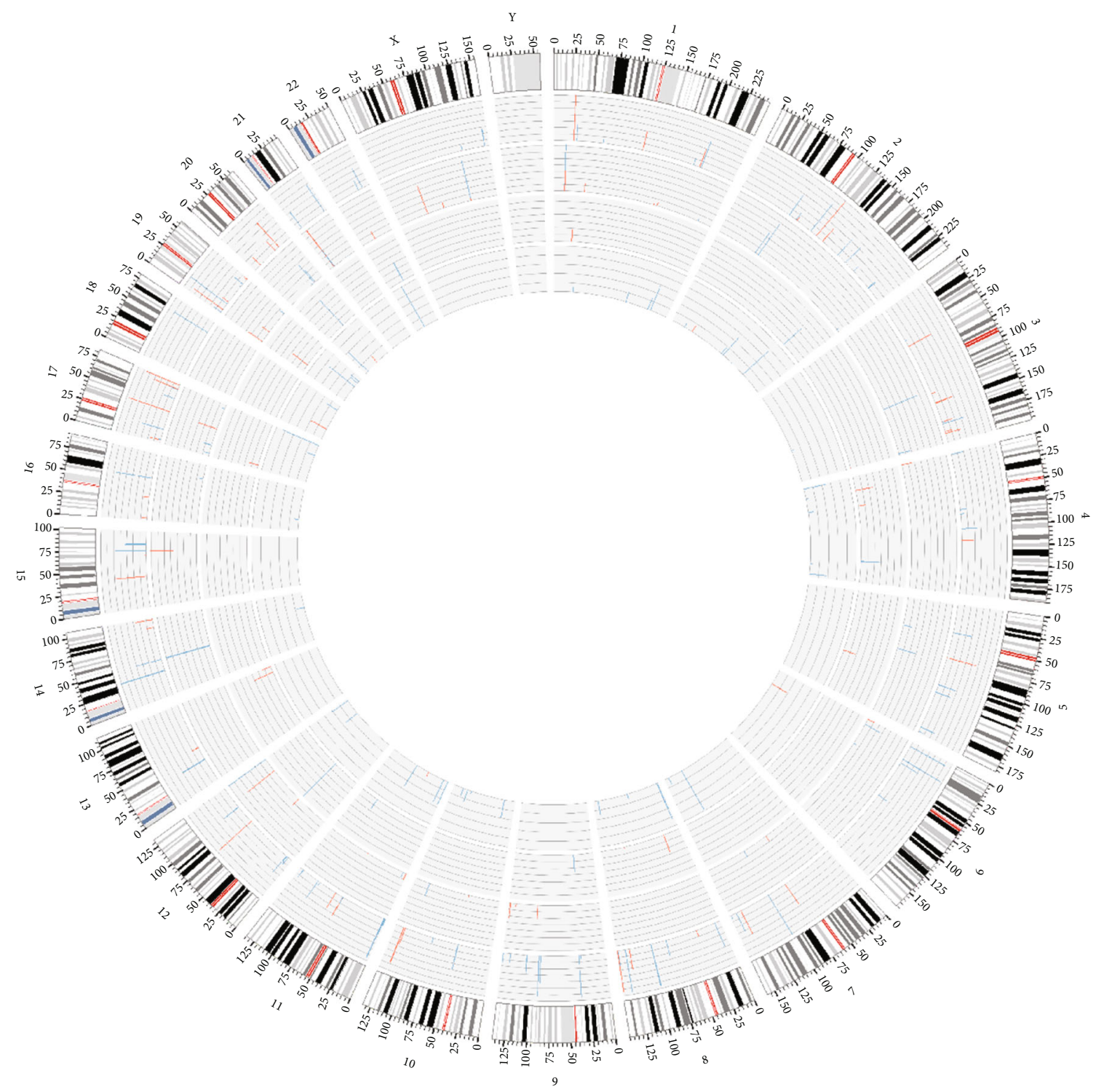

(a)

Figure 6: Continued. 


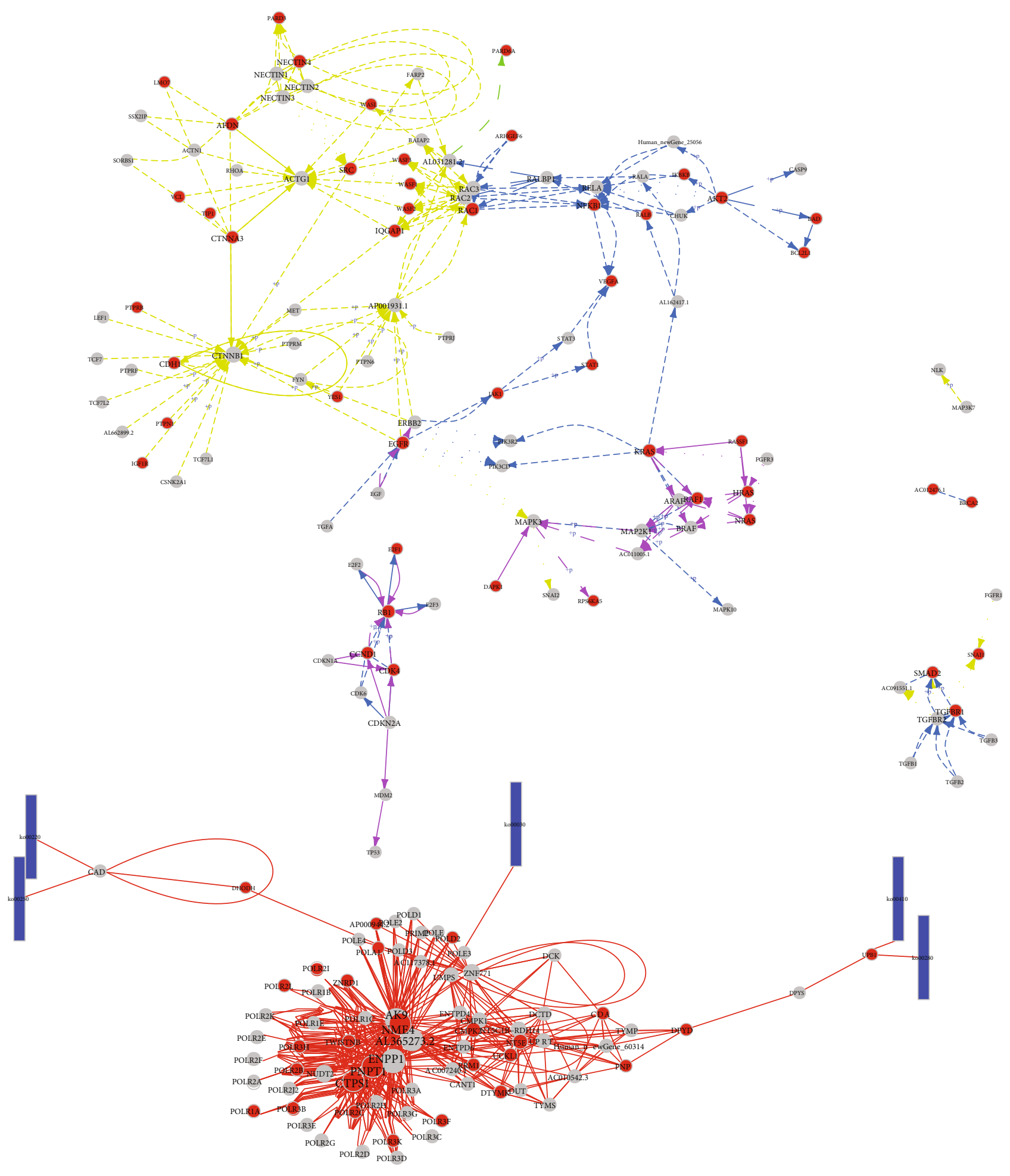

(b)

Figure 6: Continued. 


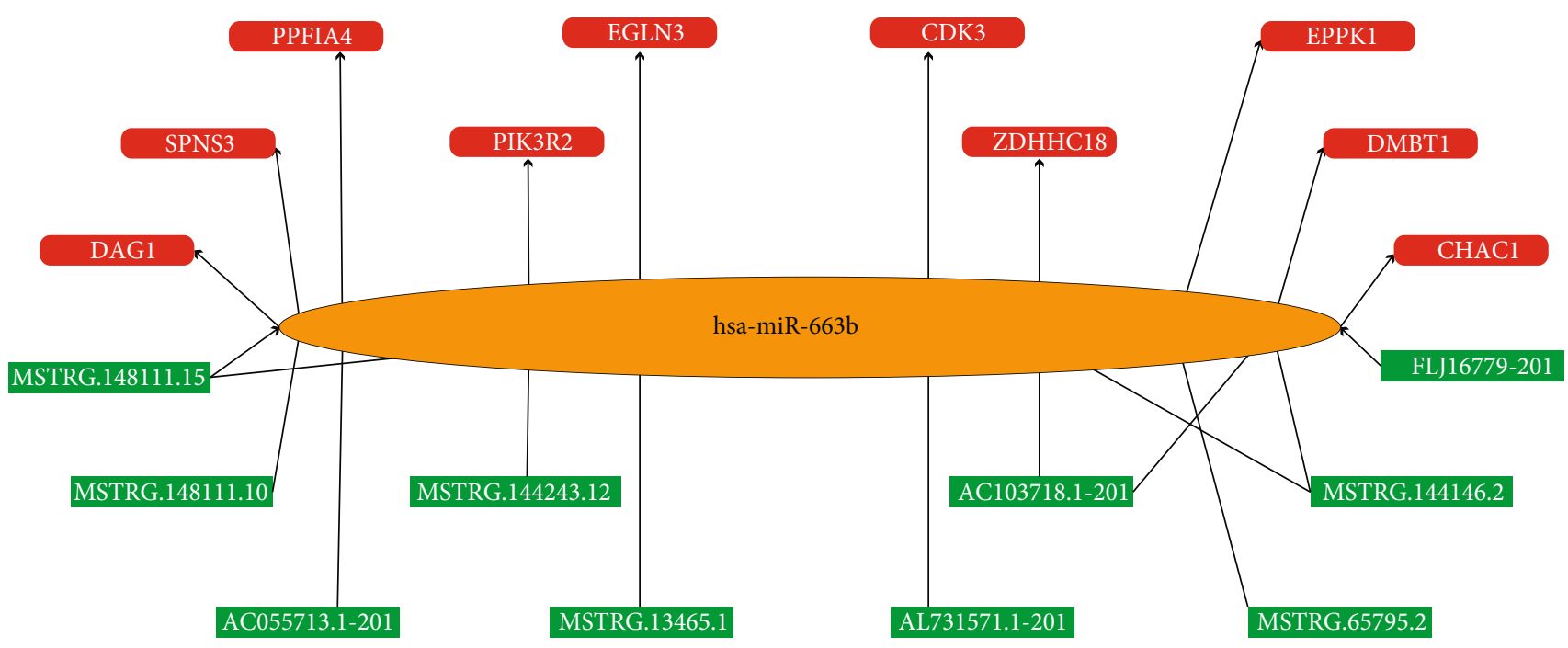

(c)

Figure 6: Combined analysis of whole transcriptome and construction of hsa-miR-663b-related ceRNA network. (a) The Circos plot of differentially expressed RNAs. The outermost circle represents chromosome information, then followed by mRNA, lncRNA, circRNA, and miRNA. Red bars indicate upregulated gene, and blue bars indicate downregulated gene. The height of bar represents significance. (b) Pathway enrichment analysis of the crucial RNAs. Dot: gene; rectangle: pathway; red dot: crucial gene; line: interrelation of gene-gene or gene-pathway. (c) Construction of hsa-miR-663b-related ceRNA network.

TAble 3: The DElncRNA-DEmiRNA-DEG ceRNA pairs.

\begin{tabular}{lcc}
\hline lncRNA & miRNA & Gene \\
\hline MSTRG.148111.15 & hsa-miR-663b & DAG1 \\
MSTRG.148111.10 & hsa-miR-663b & CHAC1 \\
FLJ16779-201 & hsa-miR-663b & CHAC1 \\
MSTRG.16479.1 & novel_miR_1799 & PIK3R2 \\
MSTRG.1512.7 & hsa-miR-663b & PIK3R2 \\
MSTRG.144243.12 & novel_miR_1799; novel_miR_752 & PIK3R2 \\
MSTRG.141087.2 & hsa-miR-663b & ZDHHC18 \\
AC103718.1-201 & hsa-miR-663b & ZDHHC18 \\
MSTRG.144146.2 & hsa-miR-663b & DMBT1 \\
AC103718.1-201 & hsa-miR-663b & DMBT1 \\
MSTRG.144146.2 & hsa-miR-663b & EGLN3 \\
MSTRG.13465.1 & hsa-miR-663b & EGLN3 \\
MSTRG.148111.15 & novel_miR_1799 & CASP14 \\
DLEU1-212 & hsa-miR-663b & CDK3 \\
AL731571.1-201 & hsa-miR-663b & PPFIA4 \\
AC055713.1-201 & hsa-miR-663b & EPPK1 \\
MSTRG.65795.2 & &
\end{tabular}

researches. LC3-I and LC3-II were the nonlipidated and lipidated forms, respectively [52]. The accumulation of LC3-II is often represented with interruption of the autophagosomelysosome fusion step [53]. SQSTM1/p62 protein was an index of autophagic degradation, and the lower levels of
SQSTM1/p62 usually represented the activation of autophagy [54]. The above results seemingly demonstrated that autophagic flux of $16 \mathrm{HBE}$ was interrupted under stimulation of $1000 \mathrm{ng} / \mathrm{ml}$ LPS. Under our observation, the amount of autophagic vacuoles was indeed increased with LPS and 
showed higher levels on the exposure of $1000 \mathrm{ng} / \mathrm{ml}$ LPS through immunofluorescent staining and electron microscopy. Generally, the initial autophagic vacuoles, also called autophagosomes, typically had a double membrane [55], whereas there was typically only one limiting membrane in the AVd, and it usually contained electron-dense cytoplasmic and/or organelles at various stages of degradation $[56,57]$. In the late digestion process, there were only a few membrane fragments and it was hard to distinguish amphisomes, autolysosomes, and lysosomes [58]. However, the most autophagic vacuoles were single-membrane and the typical double-membrane vacuoles were not significant in our research. These results may demonstrate that when exposed to $1000 \mathrm{ng} / \mathrm{ml}$ LPS, the autophagic flux of $16 \mathrm{HBE}$ was interrupted in late phage and the degradation of autophagosome may be suppressed by stimulation of LPS.

A total of 750 significant DEGs were identified, which mainly enriched in MAPK kinase kinase binding in molecular function and oxidative phosphorylation pathway. When exposed to LPS, these DEGs may play a crucial regulatory in LPS-induced ALI. Then, we constructed PPI network to visualize the interaction relationship of DEGs and selected 36 core genes to reanalyze for functional enrichment. These genes mainly enriched in 6 different GO terms. In addition, we also analyzed the targeted genes of differentially expressed lncRNA, circRNA, and miRNA for functional enrichment. It was worth to be mentioned that the targeted genes of miRNA mainly enriched in endocytosis and MAPK signaling. Multiple researches had demonstrated that MAPK signaling was one of the upstream regulatory mechanisms of mitophagy. Mangiferin could suppress PTEN-induced kinase 1 (PINK1)-parkin RBR E3 ubiquitin protein ligase (PRKN) mitophagy via protein kinase A (PKA)-MAPK signaling to promote a brown fat-like phenotype in murine C3H10T1/2 mesenchymal stem cells [59]. The sonodynamic therapy could trigger MAPK/p38-PINK1-PRKN-dependent mitophagy, in which the antioxidant suppressed the MAPK/p38 activation and sonotoxicity [60]. What is more, Chen et al. had proved that p38 MAPK could directly phosphorylate PRKN at serine 131 to disturb the protective function of mitophagy [61]. However, if mitophagy is also interrupted along with the suppression of autophagy in LPS-stimulated $16 \mathrm{HBE}$ is worth to further exploration.

ceRNA was the research focus in recent years, which was a new transcriptional regulatory mechanism. In combined analysis of several kinds of differentially expressed RNA, we selected the differentially expressed mRNA, lncRNA, circRNA, and miRNA for further analysis of ceRNA relationship. Then, we found hsa-miR-663b as a core molecule in the ceRNA network, which was the target of several regulatory ceRNA. In previous researches, hsa-miR-663b usually was described as a tumor promoter in endometrial cancer, osteosarcoma, bladder cancer, and colorectal cancer [62-65]. However, in an analysis of whole-genome miRNA expression in peripheral total blood samples of patients with acute myocardial infarction (AMI), hsa-miR-663b also showed high sensitivity and specificity for the discrepancy from the control groups, which may reveal hsa-miR-663b could be a significant biomarker for cardiovascular diseases
[66]. Interestingly, Ragusa et al. detected the miRNA transcriptome in colorectal cancer with the treatment of MAPK/extracellular signal-regulated kinase (ERK) inhibitors and found that hsa-miR-663b was upregulated in three cell lines and induced downregulation of cyclin D2 (CCND2) [67]. It may hint us that there were underlying regulatory mechanisms between hsa-miR-663b and MAPK signaling. In the next step, we were aiming to explore the expression of hsa-miR-663b in ALI and other lung diseases and then furtherly constructed and verified the hsa-miR-663b-related ceRNA regulatory network.

\section{Conclusion}

Our research constructed a novel hsa-miR-663b-related ceRNA regulatory network in LPS-induced ALI by a whole transcriptome sequencing and comprehensive bioinformatics analysis, which may contribute to the key regulatory mechanism in LPS-induced changes of autophagy and ALI. There may exist an underlying regulatory mechanism between hsa-miR-663b, MAPK signaling, and autophagy/mitophagy. Nevertheless, the expression level of hsa-miR$663 \mathrm{~b}$ and specific regulatory mechanism of ceRNA in ALI should be furtherly validated by molecular experiments.

\section{Abbreviations}

$\begin{array}{ll}\text { ALI: } & \text { Acute lung injury } \\ \text { LPS: } & \text { Lipopolysaccharide } \\ \text { 4-PBA: } & \text { 4-Phenyl butyric acid } \\ \text { NF- } \kappa \text { B: } & \text { Nuclear factor kappa-B } \\ \text { AKT: } & \text { AKT serine/threonine kinase 1 } \\ \text { mTOR: } & \text { Mammalian target of rapamycin } \\ \text { 3-MA: } & \text { 3-Methyladenine } \\ \text { ER: } & \text { Endoplasmic reticulum } \\ \text { TNF- } \alpha: & \text { Tumor necrosis factor alpha } \\ \text { IL: } & \text { Interleukin } \\ \text { LDH: } & \text { Lactate dehydrogenase } \\ \text { HPMVEC: } & \text { Human pulmonary microvascular } \\ & \text { endothelial cell } \\ \text { EtOH: } & \text { Ethyl alcohol } \\ \text { MD2: } & \text { Lymphocyte antigen 96 } \\ \text { ADM: } & \text { Adrenomedullin } \\ \text { AMPK: } & \text { Adenosine 5' }{ }^{-m o n o p h o s p h a t e-~(A M P-) ~} \\ & \text { activated protein kinase } \\ \text { ADSC: } & \text { Adipose-derived stem cell } \\ \text { ceRNA: } & \text { Competing endogenous RNA } \\ \text { lncRNA: } & \text { Long noncoding RNA } \\ \text { circRNA: } & \text { Circular RNA } \\ \text { ncRNA: } & \text { Noncoding RNA } \\ \text { miRNA: } & \text { MicroRNA } \\ \text { mRNA: } & \text { Messenger RNA } \\ \text { ULK1: } & \text { Unc-51-like autophagy activating kinase 1 } \\ \text { ATG: } & \text { Autophagy-related } \\ \text { APL: } & \text { Acute promyelocytic leukemia } \\ \text { PML: } & \text { PML nuclear body scaffold } \\ \text { RARA: } & \text { Retinoic acid receptor alpha } \\ \text { HOTAIR: } & \text { HOX transcript antisense RNA } \\ \text { PTEN: } & \text { Phosphatase and tensin homolog } \\ & \end{array}$


PHLPP: $\quad$ PH domain and leucine-rich repeat protein phosphatase 1

ARD: $\quad$ Age-related disease

SASP: $\quad$ Senescence-associated secretory phenotype

NIFK-AS1: NIFK antisense RNA 1

CCAT1: $\quad$ Colon cancer-associated transcript 1

MITA: Myocardial infarction-associated transcript

GAS5: $\quad$ Growth arrest-specific 5

UCA1: $\quad$ Urothelial cancer-associated 1

TGFB2-OT1: TGFB2 overlapping transcript 1

VEC: $\quad$ Vascular endothelial cell

CERS1: $\quad$ Ceramide synthase 1

NAT8L: $\quad$ N-Acetyltransferase 8-like

LARP1: $\quad$ La ribonucleoprotein 1, translational regulator

DElncRNA: Differentially expressed lncRNA

DEcircRNA: Differentially expressed circRNA

DEG: Differentially expressed gene

GO: $\quad$ Gene Ontology

GSEA: $\quad$ Gene set enrichment analysis

KEGG: $\quad$ Kyoto Encyclopedia of Genes and Genomes

STRING: $\quad$ Search Tool for the Retrieval of Interacting Genes

PPI: $\quad$ Protein-protein interaction

MCODE: The Molecular Complex Detection

MAP1LC3B: Microtubule-associated protein 1 light chain 3 beta

SQSTM1/p62: Sequestosome 1

EM: $\quad$ Electron microscopy

AVd or AVl: Autophagic vacuoles/autolysosomes

MAPK: $\quad$ Mitogen-activated protein kinase

MCC: $\quad$ Maximal clique centrality

ARDS: $\quad$ Acute respiratory distress syndrome

NLRP3: $\quad$ Nucleotide-binding oligomerization domain-like receptor containing pyrin domain 3

FOXO3: $\quad$ Forkhead box O3

PINK1: $\quad$ PTEN-induced kinase 1

PRKN: $\quad$ Parkin RBR E3 ubiquitin protein ligase

PKA: $\quad$ Protein kinase A

AMI: $\quad$ Acute myocardial infarction

ERK: $\quad$ Extracellular signal-regulated kinase

CCND2: $\quad$ Cyclin D2.

\section{Data Availability}

The raw data used and/or analyzed during the current study are available from the corresponding author on reasonable request.

\section{Consent}

Written informed consent for publication was obtained from all participants.

\section{Conflicts of Interest}

The authors have declared that no competing interest exists.

\section{Authors' Contributions}

Jian- $\mathrm{Yu}$ Liu was responsible for the conception, design, experiments, analysis of data, and writing. Ying-Xiao Jiang and Yi-Can Yang also helped to perform some of the experiments. Meng-Yu Zhang and Chen Huo were responsible for the analysis of data and revision of the manuscript. Yi-Qing $\mathrm{Qu}$ was responsible for the final approval of the version to be published. All authors gave the final approval of the version to be published and agree to be accountable for all aspects of the work.

\section{Acknowledgments}

This study was supported by the special project of Respiratory Clinical Medicine Research Center of Shandong Province (grant number 201912011) and the Shandong Province Medical and Health Technology Development Plan Project (grant number 2019WS082).

\section{Supplementary Materials}

Figure S1A: Gene Ontology terms of cis-targeted genes of differentially expressed lncRNAs in LPS-stimulated 16HBE in biological process. Figure S1B: Gene Ontology terms of cis-targeted genes of differentially expressed lncRNAs in LPS-stimulated 16HBE in cellular component. Figure S1C: Gene Ontology terms of cis-targeted genes of differentially expressed lncRNAs in LPS-stimulated 16HBE in molecular function. Figure S1D: the KEGG enrichment analysis of cis-targeted genes of differentially expressed lncRNAs in LPS-stimulated 16HBE. Figure S1E: Gene Ontology terms of trans-targeted genes of differentially expressed lncRNAs in LPS-stimulated 16HBE in biological process. Figure S1F: Gene Ontology terms of trans-targeted genes of differentially expressed lncRNAs in LPS-stimulated 16HBE in cellular component. Figure S1G: Gene Ontology terms of transtargeted genes of differentially expressed lncRNAs in LPSstimulated $16 \mathrm{HBE}$ in molecular function. Figure $\mathrm{S} 1 \mathrm{H}$ : the KEGG enrichment analysis of trans-targeted genes of differentially expressed lncRNAs in LPS-stimulated 16HBE. Figure S1I: Gene Ontology terms of differentially expressed circRNAs in LPS-stimulated 16HBE in biological process. Figure S1J: Gene Ontology terms of differentially expressed miRNA in LPS-stimulated $16 \mathrm{HBE}$ in cellular component. Figure S1K: Gene Ontology terms of differentially expressed miRNA in LPS-stimulated $16 \mathrm{HBE}$ in molecular function. Figure S1L: the KEGG enrichment analysis of differentially expressed miRNA in LPS-stimulated 16HBE. (Supplementary Materials)

\section{References}

[1] M. A. Matthay, L. B. Ware, and G. A. Zimmerman, "The acute respiratory distress syndrome," The Journal of Clinical Investigation, vol. 122, no. 8, pp. 2731-2740, 2012.

[2] A. P. Wheeler and G. R. Bernard, "Acute lung injury and the acute respiratory distress syndrome: a clinical review," The Lancet, vol. 369, no. 9572, pp. 1553-1564, 2007.

[3] J. L. Ather, J. F. Alcorn, A. L. Brown et al., "Distinct functions of airway epithelial nuclear Factor- $\kappa \mathrm{B}$ activity regulate 
nitrogen dioxide-induced acute lung injury," American Journal of Respiratory Cell and Molecular Biology, vol. 43, no. 4, pp. 443-451, 2010.

[4] Y. A. Mebratu and Y. Tesfaigzi, "IL-17 plays a role in respiratory syncytial virus-induced lung inflammation and emphysema in elastase and LPS-injured mice," American Journal of Respiratory Cell and Molecular Biology, vol. 58, no. 6, pp. 717-726, 2018.

[5] F. F. Stellari, A. Sala, F. Ruscitti et al., "CHF6001 inhibits NF$\kappa \mathrm{B}$ activation and neutrophilic recruitment in LPS-induced lung inflammation in mice," Frontiers in Pharmacology, vol. 10, p. 1337, 2019.

[6] Y. Zou, X. Chen, J. Xiao et al., "Neutrophil extracellular traps promote lipopolysaccharide-induced airway inflammation and mucus hypersecretion in mice," Oncotarget, vol. 9, no. 17, pp. 13276-13286, 2018.

[7] D. Zhang, J. Zhou, L. C. Ye et al., "Autophagy maintains the integrity of endothelial barrier in LPS-induced lung injury," Journal of Cellular Physiology, vol. 233, no. 1, pp. 688-698, 2018.

[8] M. Zeng, W. Sang, S. Chen et al., “4-PBA inhibits LPS-induced inflammation through regulating ER stress and autophagy in acute lung injury models," Toxicology Letters, vol. 271, pp. 26-37, 2017.

[9] T. Inui, M. Watanabe, K. Nakamoto et al., "Bronchial epithelial cells produce CXCL1 in response to LPS and TNF $\alpha$ : a potential role in the pathogenesis of COPD," Experimental Lung Research, vol. 44, no. 7, pp. 323-331, 2018.

[10] A. Hulina, M. Grdić Rajković, D. Jakšić Despot et al., "Extracellular Hsp70 induces inflammation and modulates LPS/LTA-stimulated inflammatory response in THP-1 cells," Cell Stress \& Chaperones, vol. 23, no. 3, pp. 373-384, 2018.

[11] P. Wang, X. Han, B. Mo, G. Huang, and C. Wang, "LPS enhances TLR4 expression and IFN- $\gamma$ production via the TLR4/IRAK/NF- $\kappa \mathrm{B}$ signaling pathway in rat pulmonary arterial smooth muscle cells," Molecular Medicine Reports, vol. 16, no. 3, pp. 3111-3116, 2017.

[12] B. Levine and G. Kroemer, "Biological functions of autophagy genes: a disease perspective," Cell, vol. 176, no. 1-2, pp. 11-42, 2019.

[13] X. Zhao, Z. Yu, Z. Lv et al., "Activation of alpha-7 nicotinic acetylcholine receptors $(\alpha 7 \mathrm{nAchR})$ promotes the protective autophagy in LPS-induced acute lung injury (ALI) in vitro and in vivo," Inflammation, vol. 42, no. 6, pp. 2236-2245, 2019.

[14] N. Nosaka, D. Martinon, D. Moreira, T. R. Crother, M. Arditi, and K. Shimada, "Autophagy protects against developing increased lung permeability and hypoxemia by down regulating inflammasome activity and IL- $1 \beta$ in LPS plus mechanical ventilation-induced acute lung injury," Frontiers in Immunology, vol. 11, p. 207, 2020.

[15] X. Kong, Y. Yang, L. Ren et al., "Activation of autophagy attenuates EtOH-LPS-induced hepatic steatosis and injury through MD2 associated TLR4 signaling," Scientific Reports, vol. 7, no. 1, p. 9292, 2017.

[16] J. A. Allen, T. Diemer, P. Janus, K. H. Hales, and D. B. Hales, "Bacterial endotoxin lipopolysaccharide and reactive oxygen species inhibit Leydig cell steroidogenesis via perturbation of mitochondria," Endocrine, vol. 25, no. 3, pp. 265-276, 2004.

[17] M. M. Reddy, S. V. K. Mahipal, J. Subhashini et al., "Bacterial lipopolysaccharide-induced oxidative stress in the impairment of steroidogenesis and spermatogenesis in rats," Reproductive Toxicology, vol. 22, no. 3, pp. 493-500, 2006.

[18] M. R. Metukuri, C. M. T. Reddy, P. R. K. Reddy, and P. Reddanna, "Bacterial LPS Mediated acute inflammationinduced spermatogenic failure in rats: role of stress response proteins and mitochondrial dysfunction," Inflammation, vol. 33, no. 4, pp. 235-243, 2010.

[19] M.-Y. Li, X.-L. Zhu, B.-X. Zhao et al., "Adrenomedullin alleviates the pyroptosis of Leydig cells by promoting autophagy via the ROS-AMPK-mTOR axis," Cell Death \& Disease, vol. 10, no. 7, p. 489, 2019.

[20] F. Han, Q.-Q. Xiao, S. Peng et al., "Atorvastatin ameliorates LPS-induced inflammatory response by autophagy via AKT/mTOR signaling pathway," Journal of Cellular Biochemistry, vol. 119, no. 2, pp. 1604-1615, 2018.

[21] Y. Cao, J. Chen, G. Ren, Y. Zhang, X. Tan, and L. Yang, "Punicalagin prevents inflammation in LPS-induced RAW264.7 macrophages by inhibiting FoxO3a/autophagy signaling pathway," Nutrients, vol. 11, no. 11, p. 2794, 2019.

[22] C. Bussi, J. M. Peralta Ramos, D. S. Arroyo et al., “Autophagy down regulates pro-inflammatory mediators in BV2 microglial cells and rescues both LPS and alpha-synuclein induced neuronal cell death," Scientific Reports, vol. 7, no. 1, article 43153, 2017.

[23] C. Li, J. Pan, L. Ye et al., "Autophagy regulates the therapeutic potential of adipose-derived stem cells in LPS-induced pulmonary microvascular barrier damage," Cell Death \& Disease, vol. 10, no. 11, p. 804, 2019.

[24] M. Zhou, W. Xu, J. Wang et al., "Boosting mTOR-dependent autophagy via upstream TLR4-MyD88-MAPK signalling and downstream NF- $\kappa$ B pathway quenches intestinal inflammation and oxidative stress injury," eBioMedicine, vol. 35, pp. 345-360, 2018.

[25] M. Chen, J. Liu, W. Yang, and W. Ling, "Lipopolysaccharide mediates hepatic stellate cell activation by regulating autophagy and retinoic acid signaling," Autophagy, vol. 13, no. 11, pp. 1813-1827, 2017.

[26] J.-W. Lee, H. Nam, L. E. Kim et al., “TLR4 (toll-like receptor 4) activation suppresses autophagy through inhibition of FOXO3 and impairs phagocytic capacity of microglia," Autophagy, vol. 15, no. 5, pp. 753-770, 2019.

[27] H. Zhang and B. Lu, "The roles of ceRNAs-mediated autophagy in cancer chemoresistance and metastasis," Cancers, vol. 12 , no. 10, p. 2926, 2020.

[28] L. Nie, P. Zhang, Q. Wang, X. Zhou, and Q. Wang, "lncRNAtriggered macrophage inflammaging deteriorates age-related diseases," Mediators of Inflammation, vol. 2019, Article ID 4260309, 12 pages, 2019.

[29] Y. Tay, J. Rinn, and P. P. Pandolf, “The multilayered complexity of ceRNA crosstalk and competition," Nature, vol. 505, no. 7483, pp. 344-352, 2014.

[30] F. A. Karreth and P. P. Pandolfi, "ceRNA cross-talk in cancer: when ce-bling rivalries go awry," Cancer Discovery, vol. 3, no. 10, pp. 1113-1121, 2013.

[31] Z.-H. Chen, W.-T. Wang, W. Huang et al., "The lncRNA HOTAIRM1 regulates the degradation of PML-RARA oncoprotein and myeloid cell differentiation by enhancing the autophagy pathway," Cell Death and Differentiation, vol. 24, no. 2, pp. 212-224, 2017.

[32] Y. Liu, X. Chen, X. Chen et al., "Long non-coding RNA HOTAIR knockdown enhances radiosensitivity through 
regulating microRNA-93/ATG12 axis in colorectal cancer," Cell Death \& Disease, vol. 11, no. 3, p. 175, 2020.

[33] C.-L. Chen, Y.-W. Tseng, J.-C. Wu et al., "Suppression of hepatocellular carcinoma by baculovirus-mediated expression of long non-coding RNA PTENP1 and MicroRNA regulation," Biomaterials, vol. 44, pp. 71-81, 2015.

[34] Y.-Y. Qian, K. Li, Q.-Y. Liu, and Z.-S. Liu, "Long non-coding RNA PTENP1 interacts with miR-193a-3p to suppress cell migration and invasion through the PTEN pathway in hepatocellular carcinoma," Oncotarget, vol. 8, no. 64, pp. 107859107869, 2017.

[35] N. Frey, S. Venturelli, L. Zender, and M. Bitzer, "Cellular senescence in gastrointestinal diseases: from pathogenesis to therapeutics," Nature Reviews Gastroenterology \& Hepatology, vol. 15, no. 2, pp. 81-95, 2018.

[36] J. Liu, D. Ding, Z. Jiang, T. du, J. Liu, and Z. Kong, "Long noncoding RNA CCAT1/miR-148a/PKC $\zeta$ prevents cell migration of prostate cancer by altering macrophage polarization," The Prostate, vol. 79, no. 1, pp. 105-112, 2019.

[37] Y.-X. Zhou, W. Zhao, L.-W. Mao et al., "Long non-coding RNA NIFK-AS1 inhibits M2 polarization of macrophages in endometrial cancer through targeting miR-146a," The International Journal of Biochemistry \& Cell Biology, vol. 104, pp. 2533, 2018.

[38] X. Hu, R. Ma, W. Fu, C. Zhang, and X. Du, "LncRNA UCA1 sponges miR-206 to exacerbate oxidative stress and apoptosis induced by ox-LDL in human macrophages," Journal of Cellular Physiology, vol. 234, no. 8, pp. 14154-14160, 2019.

[39] J. Liu, G.-Q. Huang, and Z.-P. Ke, "Silence of long intergenic noncoding RNA HOTAIR ameliorates oxidative stress and inflammation response in ox-LDL-treated human macrophages by upregulating miR-330-5p," Journal of Cellular Physiology, vol. 234, no. 4, pp. 5134-5142, 2019.

[40] J. Ye, C. Wang, D. Wang, and H. Yuan, "LncRBA GSA5, upregulated by ox-LDL, aggravates inflammatory response and MMP expression in THP-1 macrophages by acting like a sponge for miR-221," Experimental Cell Research, vol. 369, no. 2, pp. 348-355, 2018.

[41] Z.-M. Ye, S. Yang, Y.-P. Xia et al., "LncRNA MIAT sponges miR-149-5p to inhibit efferocytosis in advanced atherosclerosis through CD47 upregulation," Cell Death \& Disease, vol. 10, no. 2, p. 138, 2019.

[42] Z. Wang and Y. Zheng, "IncRNAs regulate innate immune responses and their roles in macrophage polarization," Mediators of Inflammation, vol. 2018, Article ID 8050956, 8 pages, 2018.

[43] S. Y. Huang, W. Lu, D. Ge et al., "A new microRNA signal pathway regulated by long noncoding RNA TGFB2-OT1 in autophagy and inflammation of vascular endothelial cells," Autophagy, vol. 11, no. 12, pp. 2172-2183, 2015.

[44] D. Szklarczyk, A. Franceschini, S. Wyder et al., "STRING v10: protein-protein interaction networks, integrated over the tree of life," Nucleic Acids Research, vol. 43, no. D1, pp. D447D452, 2015.

[45] R. Saito, M. E. Smoot, K. Ono et al., "A travel guide to Cytoscape plugins," Nature Methods, vol. 9, no. 11, pp. 1069-1076, 2012.

[46] C.-H. Chin, S.-H. Chen, H.-H. Wu, C.-W. Ho, M.-T. Ko, and C.-Y. Lin, "cytoHubba: identifying hub objects and subnetworks from complex interactome," BMC Syst Biol, vol. 8, article S11, Supplement 4, 2014.
[47] M. A. Matthay, R. L. Zemans, G. A. Zimmerman et al., "Acute respiratory distress syndrome," Nature Reviews Disease Primers, vol. 5, no. 1, p. 18, 2019.

[48] S. R. Lewis, M. W. Pritchard, C. M. Thomas, A. F. Smith, and Cochrane Emergency and Critical Care Group, "Pharmacological agents for adults with acute respiratory distress syndrome," Cochrane Database of Systematic Reviews, vol. 7, no. 7, article CD004477, 2019.

[49] Y. Hu, J. Lou, Y.-Y. Mao et al., "activation of MTOR in pulmonary epithelium promotes LPS-induced acute lung injury," Autophagy, vol. 12, no. 12, pp. 2286-2299, 2016.

[50] Y. Zhang, G. Liu, R. O. Dull, D. E. Schwartz, and G. Hu, "Autophagy in pulmonary macrophages mediates lung inflammatory injury via NLRP3 inflammasome activation during mechanical ventilation," American Journal of Physiology. Lung Cellular and Molecular Physiology, vol. 307, no. 2, pp. L173-L185, 2014.

[51] L. Song, F. Zhou, L. Cheng et al., "MicroRNA-34a suppresses autophagy in alveolar type II epithelial cells in acute lung injury by inhibiting FoxO3 expression," Inflammation, vol. 40, no. 3, pp. 927-936, 2017.

[52] D. J. Klionsky, A. K. Abdel-Aziz, S. Abdelfatah et al., "Guidelines for the use and interpretation of assays for monitoring autophagy (4th edition)," Autophagy, vol. 17, no. 1, pp. 1382, 2021.

[53] N. Engedal, M. L. Torgersen, I. J. Guldvik et al., "Modulation of intracellular calcium homeostasis blocks autophagosome formation," Autophagy, vol. 9, no. 10, pp. 1475-1490, 2013.

[54] J.-H. Lee, W. H. Yu, A. Kumar et al., "Lysosomal proteolysis and autophagy require presenilin 1 and are disrupted by Alzheimer-related PS1 mutations," Cell, vol. 141, no. 7, pp. 1146-1158, 2010.

[55] E.-L. Eskelinen and A. L. Kovács, "Double membranes vs. lipid bilayers, and their significance for correct identification of macroautophagic structures," Autophagy, vol. 7, no. 9, pp. 931-932, 2011.

[56] P. Ylä-Anttila, H. Vihinen, E. Jokitalo, and E.-L. Eskelinen, "Chapter 10 Monitoring Autophagy by Electron Microscopy in Mammalian Cells," Methods in Enzymology, vol. 452, pp. 143-164, 2009.

[57] E.-L. Eskelinen, "Fine structure of the autophagosome," Methods in Molecular Biology, vol. 445, pp. 11-28, 2008.

[58] E.-L. Eskelinen, F. Reggiori, M. Baba, A. L. Kovács, and P. O. Seglen, "Seeing is believing: the impact of electron microscopy on autophagy research," Autophagy, vol. 7, no. 9, pp. 935-956, 2011.

[59] M. S. Rahman and Y.-S. Kim, "PINK1-PRKN mitophagy suppression by Mangiferin promotes a brown-fat-phenotype via PKA-p38 MAPK signalling in murine C3H10T1/2 mesenchymal stem cells," Metabolism, vol. 107, article 154228, 2020.

[60] F. Qu, P. Wang, K. Zhang et al., "Manipulation of mitophagy by "all-in-one" nanosensitizer augments sonodynamic glioma therapy," Autophagy, vol. 16, no. 8, pp. 1413-1435, 2020.

[61] J. Chen, Y. Ren, C. Gui et al., "Phosphorylation of Parkin at serine 131 by $\mathrm{p} 38$ MAPK promotes mitochondrial dysfunction and neuronal death in mutant A53T $\alpha$-synuclein model of Parkinson's disease," Cell Death \& Disease, vol. 9, no. 6, p. 700, 2018.

[62] Y.-L. Wang, Y. Shen, J.-P. Xu et al., "Pterostilbene suppresses human endometrial cancer cells in vitro by down- regulating 
miR-663b," Acta Pharmacologica Sinica, vol. 38, no. 10, pp. 1394-1400, 2017.

[63] Y. Shu, W. Ye, Y. L. Gu, and P. Sun, "Blockade of miR-663b inhibits cell proliferation and induces apoptosis in osteosarcoma via regulating TP73 expression," Bratislavské Lekárske Listy, vol. 119, no. 1, pp. 41-46, 2018.

[64] X. Yin, X. Zheng, M. Liu et al., "Exosomal miR-663b targets Ets2-repressor factor to promote proliferation and the epithelial-mesenchymal transition of bladder cancer cells," Cell Biology International, vol. 44, no. 4, pp. 958-965, 2020.

[65] S. Hong, Z. Yan, H. Wang, L. Ding, Y. Song, and M. Bi, “miR$663 \mathrm{~b}$ promotes colorectal cancer progression by activating Ras/Raf signaling through downregulation of TNK1," Human Cell, vol. 33, no. 1, pp. 104-115, 2020.

[66] B. Meder, A. Keller, B. Vogel et al., "MicroRNA signatures in total peripheral blood as novel biomarkers for acute myocardial infarction," Basic Research in Cardiology, vol. 106, no. 1, pp. 13-23, 2011.

[67] M. Ragusa, L. Statello, M. Maugeri et al., "Specific alterations of the microRNA transcriptome and global network structure in colorectal cancer after treatment with MAPK/ERK inhibitors," Journal of Molecular Medicine (Berlin, Germany), vol. 90, no. 12, pp. 1421-1438, 2012. 